\title{
Adhesive Hemostatic Conducting Injectable Composite Hydrogels with Sustained Drug Release and Photothermal Antibacterial Activity to Promote Full-Thickness Skin Regeneration During Wound Healing
}

\author{
Yongping Liang, Xin Zhao, Tianli Hu, Baojun Chen, Zhanhai Yin, Peter X. Ma, \\ and Baolin Guo*
}

Developing injectable nanocomposite conductive hydrogel dressings with multifunctions including adhesiveness, antibacterial, and radical scavenging ability and good mechanical property to enhance full-thickness skin wound regeneration is highly desirable in clinical application. Herein, a series of adhesive hemostatic antioxidant conductive photothermal antibacterial hydrogels based on hyaluronic acid-graft-dopamine and reduced graphene oxide ( $\mathrm{rCO}$ ) using a $\mathrm{H}_{2} \mathrm{O}_{2} / \mathrm{HPR}$ (horseradish peroxidase) system are prepared for wound dressing. These hydrogels exhibit high swelling, degradability, tunable rheological property, and similar or superior mechanical properties to human skin. The polydopamine endowed antioxidant activity, tissue adhesiveness and hemostatic ability, self-healing ability, conductivity, and NIR irradiation enhanced in vivo antibacterial behavior of the hydrogels are investigated. Moreover, drug release and zone of inhibition tests confirm sustained drug release capacity of the hydrogels. Furthermore, the hydrogel dressings significantly enhance vascularization by upregulating growth factor expression of CD31 and improve the granulation tissue thickness and collagen deposition, all of which promote wound closure and contribute to a better therapeutic effect than the commercial Tegaderm films group in a mouse full-thickness wounds model. In summary, these adhesive hemostatic antioxidative conductive hydrogels with sustained drug release property to promote complete skin regeneration are an excellent wound dressing for fullthickness skin repair.

\section{Introduction}

In human body, skin tissue is the largest, most exposed, and also most vulnerable tissue..$^{[1,2]}$ Once the damage of skin tissue occurs, the repair process appears to be very complex, ${ }^{[3-5]}$ which includes four interactive and classic phases: hemostasis, inflammation, proliferation, and remodeling/maturation. ${ }^{[6]}$ Although most skin defect can be quickly and effectively healed within 1 or 2 weeks,,${ }^{[7,8]}$ the extensive full-thickness wounds are often hard to repair, which presents serious impact on health and even threaten people's life. ${ }^{\left[{ }^{9]}\right.}$ Therefore, a variety of biomaterials have been developed as wound dressings, such as nanofibers, sponge, foam, and hydrogels. ${ }^{[10,11]}$ Among these materials, injectable polymeric hydrogels have become a promising option due to their soft properties similar to extracellular matrix (ECM), adjustable physical and chemical properties, and ability to fill any irregular shaped wound. ${ }^{[12]}$ Especially, injectable hydrogels with adhesiveness can attach and bond the defected tissues together, which accelerate the wound
Y. Liang, Dr. X. Zhao, T. Hu, Prof. B. Guo

Frontier Institute of Science and Technology, and State Key Laboratory

for Mechanical Behavior of Materials

Xi'an Jiaotong University

Xi'an 710049, China

E-mail: baoling@mail.xjtu.edu.cn

B. Chen, Prof. Z. Yin

Department of Orthopaedics

The First Affiliated Hospital

College of Medicine

Xi'an Jiaotong University

Xi'an 710061, China

The ORCID identification number(s) for the author(s) of this article can be found under https://doi.org/10.1002/smll.201900046.
Prof. P. X. Ma

Macromolecular Science and Engineering Center

University of Michigan

Ann Arbor, MI 48109, USA

Prof. P. X. Ma

Department of Materials Science and Engineering

University of Michigan

Ann Arbor, MI 48109, USA

Prof. B. Guo

Key Laboratory of Shaanxi Province for Craniofacial Precision

Medicine Research

College of Stomatology

Xi'an Jiaotong University

Xi'an 710049, China

DOI: $10.1002 / \mathrm{smll} .201900046$ 
heal properly. Besides, it can also play the role of hemostat or sealant for stopping the bleeding or preventing the leakage of fluid or gas from the wound, and serve as a shield to avoid bacterial infections. ${ }^{[13-15]}$ Thus, developing new injectable adhesive hydrogel dressing to promote the whole wound healing process is highly desirable.

Hyaluronic acid (HA), as a natural polysaccharide produced by fibroblasts in the wound repair stages of proliferation, ${ }^{[6]}$ can mediate cellular signaling, ${ }^{[16]}$ promote cell migration, ${ }^{[17]}$ and induce morphogenesis and matrix organization, which enhances the repair process of soft tissue wound. ${ }^{[18-20]}$ Besides, HA has good biocompatibility, ${ }^{[21]}$ biodegradability, ${ }^{[22]}$ moisture retention, and gelation property, ${ }^{[23]}$ which favors the design of injectable hydrogel dressing. However, although HA-based hydrogel can fill the tissue surrounding the fresh wound, ${ }^{24]}$ absorb wound extract, and reduce inflammation, it presents weak adhesive property to close the wound site. Polydopamine based or catechol contained hydrogels often possess good wet adhesiveness to soft tissues, due to the chemical crosslinking and physical bonding between polydopamine or catechol group and soft tissue. ${ }^{[25]}$ Thus, grafting dopamine (DA) to HA (hyaluronic acid-graft-dopamine (HA-DA)) will endow HA with good tissue adhesion property. ${ }^{[26,27]}$ Moreover, the introduction of catechol onto HA will also improve the hemostatic effect of HA-DA-based hydrogel for its enhanced adhesiveness of HA-DA to the wounded tissue. Furthermore, the catechol group can endow HA-DA-based hydrogel with good antioxidant ability, which will be beneficial to accelerate the wound repair process by balancing oxidative pressure in wound area. Thus, grafting DA to HA will endow HA-DA-based hydrogels with good adhesiveness, hemostatic ability, and antioxidant property, which makes HA-DA-based hydrogels excellent candidates as multifunctional injectable hydrogel dressing.

The process of wound healing depends on the mutual effect between cytokines, growth factors, macrophages, and so on. All these activities rely on the delivery of signals that caused the proliferation of cell lines at the edges of wound, which then formed a new matrix in the gap between wounds. ${ }^{[28,29]}$ Our previous researches have also proved the promoting effect of biomaterials with conductivity properties in wound healing process. ${ }^{[30-35]}$ Graphene is a promising candidate material for its excellent electrical conductivity, biocompatibility, high surface area, and mechanical strength. ${ }^{[36]}$ Incorporation of graphene into HA-DA hydrogel can result in conductive HA-DA hydrogel dressing, which may favor to enhance the wound healing process. The high specific surface area also exhibits peculiar interactional pattern with biomolecules, cells, and even human tissues to improve the hydrogel's bioactivity. ${ }^{[36,37]}$ However, the disadvantage of poor dispersity seriously limits graphene's application. ${ }^{[38]}$ Graphene oxide (GO) shows better water dispersity due to its hydrophilic groups, such as hydroxyl group, epoxy group, and carboxyl group. But its conductivity is much lower compared to graphene. Fortunately, at a weak alkaline $\mathrm{pH}$, dopamine can serve as a reducing agent for GO to form reduced GO (rGO) to improve its conductivity, and will simultaneously undergo self-polymerization to generate a hydrophilic polydopamine (PDA) coating on the surfaces of rGO to enhance its water dispersity. ${ }^{[39,40]}$ Moreover, the obtained rGO not only presents good dispersity and good electrical conductivity, but also possesses excellent photothermal antibacterial property and bioactivity from polydopamine, all of which contribute to preparing bioactive wound dressing. The reactive polydopamine coating from $\mathrm{rGO}$ can also serve as nanofiller to reinforce the HA-DA-based hydrogel network. Thus, developing rGO and HA-DA-based nanocomposite adhesive hemostatic anti-oxidant conductive hydrogel dressing is highly anticipated in wound healing field, which has not been reported.

In this study, taking advantages of the unique functions of $\mathrm{HA}, \mathrm{DA}$, and $\mathrm{rGO}$, a series of adhesive antioxidative hemostatic conductive hydrogels were synthesized based on HA-DA and polydopamine-coated $\mathrm{rGO}$ through the oxidative coupling of catechol groups by $\mathrm{H}_{2} \mathrm{O}_{2} / \mathrm{HRP}$ catalytic system. These HA-DA/rGO hydrogels exhibited high in vitro swelling, suitable degradation, and tunable rheological properties and excellent compressible and tensile properties. The performance of tissue adhesiveness, conductivity, antioxidant activity, hemostatic ability, photothermal effect, and NIR irradiation enhanced in vitro and in vivo antibacterial behaviors were systematically investigated. Besides, more than 10 days' drug release study and zone of inhibition test showed the drug sustained release capacity of these hydrogels. The good blood compatibility and cytocompatibility were also demonstrated in vitro to confirm the hydrogel's biocompatibility. Furthermore, wound closure, collagen metabolism, histomorphological examinations, and immunofluorescence staining were carried out to evaluate the good promoting effects of these hydrogels in a mouse full-thickness wounds repair model. In summary, all of these results demonstrate the enormous potential of these adhesive conductive hemostatic hydrogels with excellent mechanical properties, antioxidant activity, photothermal effect, drug sustained release ability, and biocompatibility for wound healing application.

\section{Results and Discussion}

\subsection{Preparation of HA-DA/rGO Hydrogel}

A series of adhesive hemostatic conductive hydrogels with suitable mechanical properties, conductivity, antioxidant activity, photothermal effect, drug sustained release ability, and biocompatibility were prepared and evaluated for wound healing application in this study. The overall strategy to develop HA-DA/rGO hydrogels for wound healing is shown in Figure 1a-c. HA was selected as the main component of the hydrogels on account of its characteristics of improving skin nutrition metabolism, making skin tender, smooth, wrinkle free, increasing elasticity, and enhancing skin penetration. ${ }^{[20]}$ To endow HA with good adhesiveness and anti-oxidant property, DA was linked to HA by the classic 1-(3-dimethylaminopropyl)-3-ethylcarbodiimide hydrochloride (EDC)/N-hydroxysuccinimide (NHS) chemistry to synthesize HA-DA (Figure 1a). The ${ }^{1} \mathrm{H}$ NMR analysis results (Figure S1, Supporting Information) confirmed the successful conjugation of dopamine to HA. To improve water dispersibility and conductivity of GO, DA was coated on the surface of GO to enhance the hydrophilicity and to reduce GO in an alkaline condition (Figure 1b), and the successful preparation of rGO@PDA was confirmed through Fourier transform infrared spectroscopy (FT-IR) (Figure S2, Supporting Information) and 


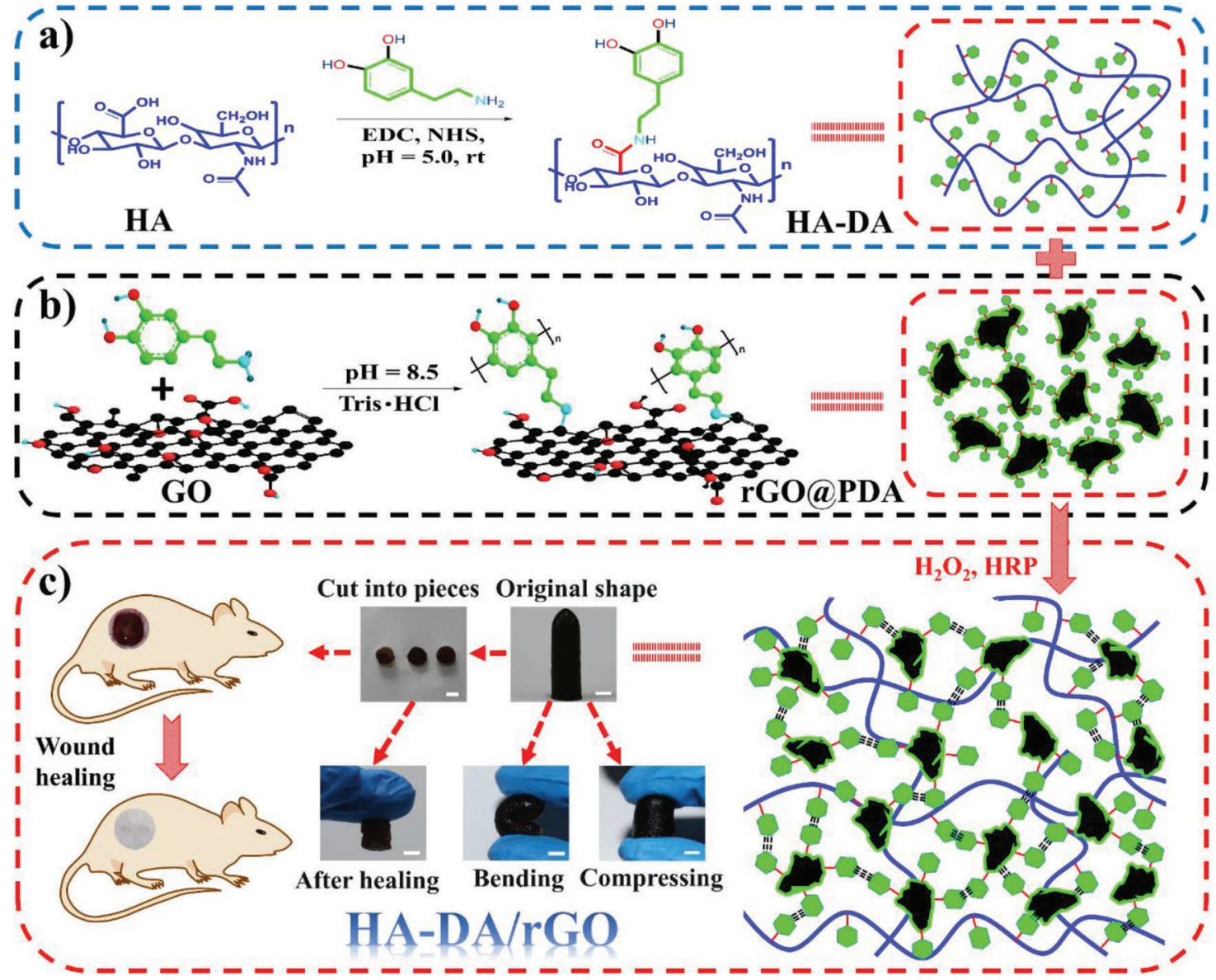

Figure 1. Diagrammatic sketch of HA-DA/rGO hydrogel preparation. a) Preparation scheme of HA-DA polymer and b) rGO@PDA, c) scheme of $\mathrm{HA}-\mathrm{DA} / \mathrm{rGO}$ hydrogel and the original, bending, compressing, self-healing representation, and the application in wound healing. Scale bar: $5 \mathrm{~mm}$.

thermogravimetric analysis (TGA) test (Figure S3, Supporting Information). As shown in Figure 1c, the HA-DA polymer chains were cross-linked with rGO@PDA by oxidative coupling of catechol groups between both of them by using $\mathrm{H}_{2} \mathrm{O}_{2}$ /HRP as an initiator system, leading to the formation of hydrogels network. To validate the crosslinking reaction in the hydrogel system, hydrogel HA-DA/GO5 was prepared where rGO@PDA was replaced with GO in the system, and the compression test was performed after the complete gel formation. The HA-DA/GO5 hydrogel $(18.3 \pm 1.9 \mathrm{kPa})$ showed significantly lower compression stress than that of HA-DA/ rGO5 hydrogel $(37.9 \pm 0.7 \mathrm{kPa})$ at a strain of $60 \%$ (Figure S4, Supporting Information), because the chemical crosslinking between rGO@PDA and HA-DA greatly enhanced the compression stress of the HA-DA/rGO5, indicating that rGO@PDA was chemically crosslinked with HA-DA within the hydrogels. Four groups of HA-DA/rGO hydrogels were synthesized by increasing the mass ratio of rGO@PDA to HA-DA from 0 to 1, 3, and $5 \mathrm{wt} \%$ and named as HA-DA/rGO0, HA-DA/rGO1, HA-DA/rGO3, and HA-DA/rGO5, respectively. The original, bending, compressing shapes of HA-DA/rGO3 hydrogels exhibited their flexible mechanical properties. When the hydrogels were cut into pieces, they could adhere together, indicating their self-healing property. Upon application, after adhering to the wound site, the self-healing capacity of these adhesive hemostatic hydrogels can ensure good performance to prevent fluid leakages, as well as bacterial penetration and infection under the circumstances of deformation or fracture of hydrogels due to external force.

\subsection{Gelation Time, Swelling, Degradation, Morphology, and Rheological Property}

For the gelation time test, the intersection of the storage modulus and loss modulus curves which derived from the rheological test is considered to be the gelation point and each group was tested at least three times. As a result, gelation time was found to be dependent on the weight ratio of rGO@PDA in this system. By using 0 wt\% rGO@PDA, the gelling process took the longest time of $557.4 \pm 51.2 \mathrm{~s}$, and the gelation time gradually decreased to $386.6 \pm 46.6,267.0 \pm 20.2$, and $157.7 \pm 33.8 \mathrm{~s}$, when the weight ratio of rGO@PDA was increased to 1, 3, and $5 \mathrm{wt} \%$, respectively. These results indicated that the higher concentration of the rGO@PDA in the hydrogel networks improves the efficiency of gelation.

The water uptake ability of hydrogel is beneficial to accelerate wound healing process by absorbing wound effluence 

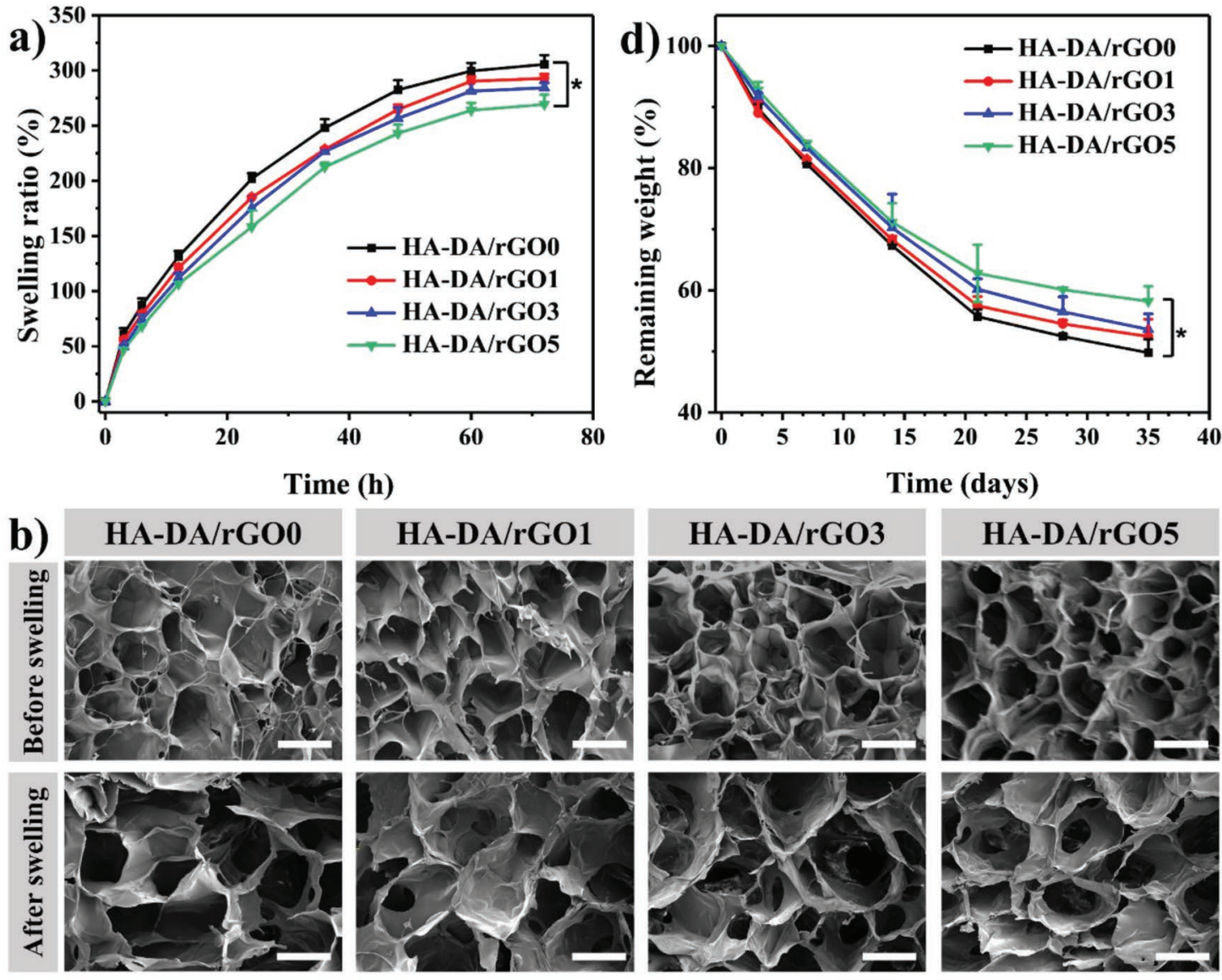

c)

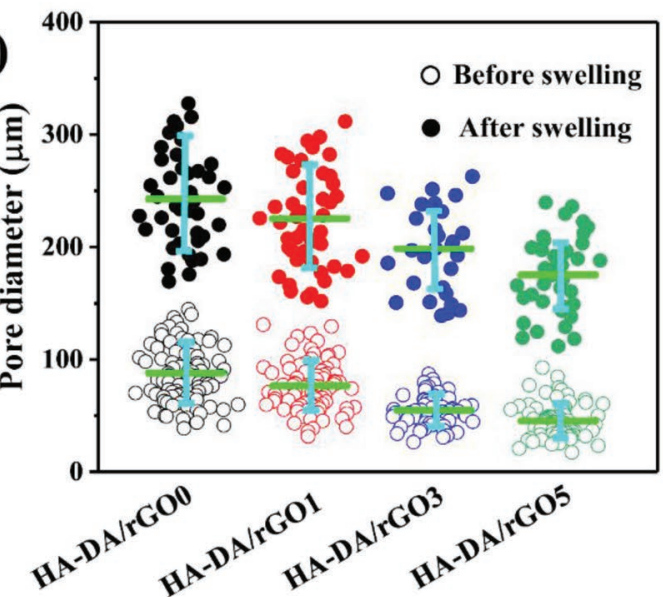

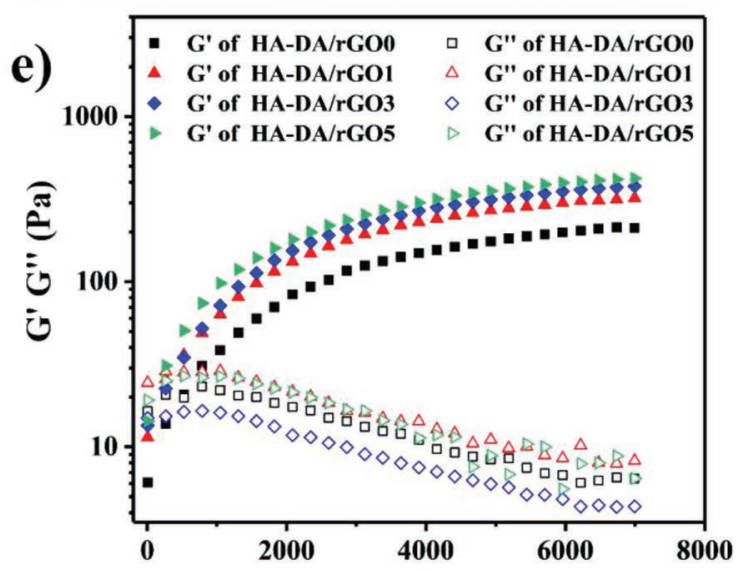

Time (s)

Figure 2. Characterization of HA-DA/rGO hydrogels. a) Swelling ratio of hydrogels in PBS ( $\mathrm{pH} 7.4)$ at $37^{\circ} \mathrm{C}$; b) SEM images of hydrogels, scale bar: $100 \mu \mathrm{m}$; c) Pore size distribution of hydrogels; d) Degradation profiles of the hydrogels in PBS with pH 7.4 at $37^{\circ} \mathrm{C}$; e) Rheological behavior of hydrogels.

and reducing the probability of infection. Swelling behaviors of these hydrogels were shown in Figure 2a. Overall, all of hydrogels reached a maximum water absorption $\approx 300 \%$ of their initial weight after 3 d. The lowest water absorption of about $269.2 \%$ was observed for HA-DA/rGO5 hydrogel over $3 \mathrm{~d}$, and $284.3 \%$, $292.9 \%$, and $305.5 \%$ for HA-DA/rGO3, HA-DA/rGO1, and $\mathrm{HA}-\mathrm{DA} / \mathrm{rGO}$, respectively, indicating that the swelling ratio increased with decreasing the concentration of rGO@PDA.
Surface morphology of these HA-DA/rGO hydrogels was observed with scanning electron microscope (SEM). The collected images and statistical results of pore diameter showed homogeneous pores with round shape (Figure $2 \mathrm{~b}$ ). The pore size (Figure 2c) of the hydrogel showed an obvious dependence on the weight ratio of rGO@PDA, that is, higher mass ratio of rGO@PDA, smaller pore size of these hydrogels. Specifically, the pore sizes for HA-DA/rGO0, HA-DA/rGO1, HA-DA/rGO3, 
and HA-DA/rGO5 hydrogels varied from $88.1 \mu \mathrm{m}$ to $76.7,54.9$, and $45.4 \mu \mathrm{m}$, respectively. Moreover, after swelling, the pore sizes still showed a dependence on the content of rGO@PDA and increased to 243.5, 225.6, 198.9, and $175.6 \mu \mathrm{m}$, respectively. Additionally, the integrity and interconnectivity of the pore structure also indicated the good structural stability of hydrogels.

Suitable degradation rate of biomaterials is also important for biomedical applications. ${ }^{[30,41-43]}$ By adjusting the concentration of rGO@PDA, the degradation rate was also proved to be tunable. The fastest degradation ratio of $50.2 \%$ was found for HA-DA/rGO0 hydrogel after about one month (Figure 2d). With the increase of rGO@PDA concentration, weight remaining ratio increased from $52.4 \%$ to $53.6 \%$ and $58.2 \%$ for HA-DA/rGO1, HA-DA/rGO3 and HA-DA/rGO5 hydrogels, respectively. These swelling and degradation results exhibited that higher mass ratio of rGO@PDA in the hydrogel networks improves the efficiency of cross-linking and stability.

To analyze the influence of different rGO@PDA weight ratio on the rheological properties of HA-DA/rGO hydrogel, the curves of storage modulus $\left(G^{\prime}\right)$ and loss modulus $\left(G^{\prime \prime}\right)$ of these hydrogels over time were recorded. $G^{\prime}$ of hydrogel with rGO@PDA weight ratio of 5 wt\% (421.5 Pa) was higher than samples with rGO@PDA weight ratio of 3 wt\% (378.7 Pa), $1 \mathrm{wt} \%(321.5 \mathrm{~Pa})$, and $0 \mathrm{wt} \%(210.9 \mathrm{~Pa})$. This is because more rGO@PDA in the system increased the crosslinking density leading to the higher $G^{\prime}$ of these hydrogels (Figure 2e).

The self-healing behavior of hydrogels was measured by rheology test. From the results of strain amplitude sweep of the HA-DA/rGO3 hydrogel, its network collapsed when the strain was $1537.8 \%$. The continuous step strain test was then used to perform the rheological recovery behavior of the hydrogel (Figure S5, Supporting Information). After the first high strain $(2000 \%)$, the $G^{\prime}$ of the recovered hydrogels decreased significantly from 300 to $5 \mathrm{~Pa}$, and $G^{\prime \prime}>G^{\prime}$, indicating the collapse of the hydrogel network. When at low strain $(1 \%)$, the $G^{\prime}$ of the hydrogel returned to $125 \mathrm{~Pa}$, indicating that crosslinking of hydrogel was partially recovered. And after experiencing another four high-low strain cycles tests, the healed hydrogel displayed nearly the same values of $G^{\prime}$ and $G^{\prime \prime}$ with the second cycle, demonstrating the self-healing of HA-DA/rGO hydrogel, probably due to the physical bonding between rGO@PDA and HA-DA including hydrogen bonding, and $\pi-\pi$ stacking. ${ }^{[4]}$

\subsection{Mechanical Properties of HA-DA/rGO Hydrogel}

Suitable mechanical properties similar to the skin is good for skin wound repairing material, which can not only maintain integrity of the material but also beneficial to good adhesion when the skin tissue is deformed by external force. ${ }^{[45]}$ The tensile testing results of these four hydrogels can be seen in Figure 3a. These hydrogels exhibited good stretchability (93.9-200.8\%), which is better than the extensibility of the human skin (60-75\%). ${ }^{[46]}$ With the addition of rGO@PDA from 0 to $5 \mathrm{wt} \%$, it is obvious that the elongation at break increased from $93.9 \%$ to $153.1 \%, 194.4 \%$ and $200.8 \%$, respectively. HA-DA/rGO5 with the highest rGO@PDA content exhibited the highest fracture strain (about 200.8\%). The tensile stress of hydrogel also increased from 18.6 to $30.9,38.4$ and $65.1 \mathrm{kPa}$, which is comparable to human skin. ${ }^{[47]}$

Compression stress-strain measurements were carried out to evaluate the mechanical properties of these hydrogels. The results in Figure 3b exhibited that incorporating rGO@ PDA into these HA-DA/rGO hydrogels could effectively enhance the mechanical properties, and hydrogel containing more rGO@PDA showed higher stress at the same strain. Specifically, the stress increased from 16.8 to 21.9, 30.5, and $36.5 \mathrm{kPa}$ at the strain of about $60 \%$ along with the increase of rGO@PDA weight ratio from 0 to 1,3 , and 5 wt $\%$, respectively. Besides, in order to further evaluate the recovery and robustness of the interconnected porous hydrogels, 50 cycles were carried out through a loading-unloading compression test by applying a strain of $60 \%$ (Figure $3 c-f$ ). After 50 cycles of loading-unloading test, the recovered hydrogels still presented similar stress-strain curves to that of original ones, demonstrating that the hydrogel has good fatigue resistance. Therefore, these hydrogels with similar or superior mechanical properties to human skin can resist external forces without fracture and better avoid the damage of potential tissues.

\subsection{Conductivity and Antioxidant Ability of HA-DA/rGO Hydrogel}

Based on the higher conductivity of rGO than graphene oxide, it was chosen as a component to prepare these HA-DA/rGO hydrogels. It has been reported that the conductive materials could accelerate the wound healing process. ${ }^{[48-52]}$ Therefore, the conductivity of these hydrogels was tested. The dried HA-DA/rGO0 hydrogel presented the lowest conductivity of $0.3 \times 10^{-4} \mathrm{~S} \mathrm{~m}^{-1}$. After the addition of rGO@PDA, conductivity of dry hydrogels were greatly improved from $1.2 \times 10^{-4}$ to $2.0 \times 10^{-4}$ and $2.5 \times 10^{-4} \mathrm{~S} \mathrm{~m}^{-1}$ for the HA-DA/rGO1, HA-DA/ rGO3, and HA-DA/rGO5 hydrogel, respectively, which mainly derived from reduced graphene's electronic conductivity (Figure 3g). Furthermore, when measurements were applied to wet hydrogels, the conductivity of HA-DA/rGO dramatically increased to the level of about $0.5 \mathrm{~S} \mathrm{~m}^{-1}$, which mainly derived from hyaluronic acid's ionic conductivity. In conclusion, the enhanced conductivity would be helpful for transmitting electrical signals from living biological tissue and promoting wound healing process.

The existence of free radicals in wound site can lead to oxidative stress, which will further result in the peroxidation of lipid, damage of DNA, and inactivation of enzyme. ${ }^{[53]}$ Studies of the topical application of materials with free radical scavenging properties on patients or animals have been demonstrated to significantly improve wound healing. ${ }^{[54]}$ The antioxidant ability of these HA-DA/rGO hydrogels was evaluated by detecting their efficiency for scavenging the stable free radical DPPH and the HA-DA/rGO3 hydrogel was chosen as a representation. As is shown in Figure $3 \mathrm{i}, 2.5 \mathrm{mg} \mathrm{mL} \mathrm{mL}^{-1}$ of the copolymer can clear almost $70 \%$ of free radicals and the antioxidant efficiency also increased to more than $90 \%$ when the content of hydrogel reached to $\geq 5 \mathrm{mg} \mathrm{mL}^{-1}$. Overall, the good antioxidant capacity of HA-DA/rGO hydrogels increases their potential application for skin wound dressing. 

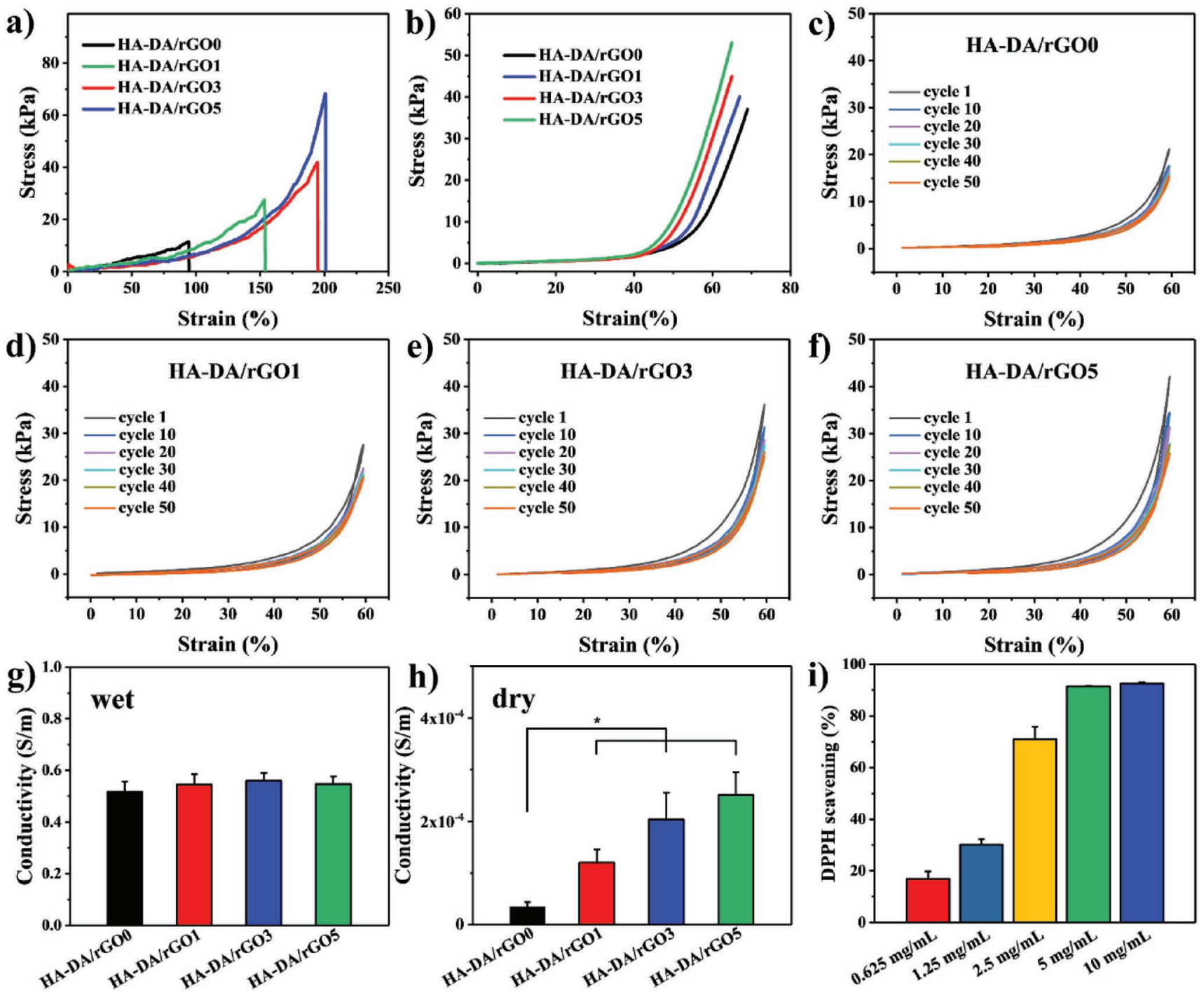

Figure 3. Mechanical characterizations, conductivity and antioxidant ability of HA-DA/rGO hydrogels. a) The stress-strain profiles about the hydrogels though tensile tests; b) Stress-strain profiles of these hydrogel by compression; The cycling curves of stress-strain for the hydrogel c) HA-DA/rGO0, d) $\mathrm{HA}-\mathrm{DA} / \mathrm{rGO} 1$, e) $\mathrm{HA}-\mathrm{DA} / \mathrm{rGO}$, and f) $\mathrm{HA}-\mathrm{DA} / \mathrm{rGO} 5$ with the compression strains of $60 \%$. Conductivity of the hydrogels at g) wet state and h) dry state; i) DPPH scavenging percentage by $\mathrm{HA}-\mathrm{DA} / \mathrm{rGO} 3$ hydrogels with different concentrations.

\subsection{Adhesion and In Vivo Hemostatic Performance of HA-DA/rGO Hydrogel}

As an excellent skin wound repair material, in addition to the good promoting effect of the ingredients on the wound repair process, good adhesion performance is also necessary. ${ }^{[13]}$ In this work, the lap-shear test was performed to estimate the adhesion capacity of these material to skin tissue (Figure 4a,b). ${ }^{[5]}$ All the hydrogels showed good adhesive strength between $5.0 \pm 0.5$ and $6.3 \pm 1.2 \mathrm{kPa}$ and had no significant difference $(P>0.05)$, which are similar with or even better than the commercial dressings (about $5 \mathrm{kPa}$ ), ${ }^{[56]}$ exhibiting that the our HA-DA/rGO hydrogels possessed great potential in wound repair. About the adhesion mechanism of these hydrogels, the imide formation or Michael-type reaction of catechol and quinone groups on rGO@PDA and HA-DA with amino or thiol groups on the protein should be a reasonable explanation. ${ }^{[57]}$

In skin trauma, especially in full-thickness skin defect, bleeding was inevitable. Whether the bleeding could be stopped rapidly acted as a vital role in the first stage of repair. Based on the good tissue adhesiveness of $\mathrm{HA}-\mathrm{DA} / \mathrm{rGO}$ hydrogel, the hydrogels could be used as hemostat. ${ }^{[4]}$ Hence, a liver bleeding mouse model was established to confirm the hemostatic ability of HA-DA/rGO hydrogel. In Figure 4c, the control group reached to about $398.3 \pm 60.1 \mathrm{mg}$ outflow of blood from the mouse' liver while only $74.2 \pm 36.2 \mathrm{mg}$ was presented in the HA-DA/rGO3 hydrogel group, and the blood loss between control group and HA-DA/rGO3 hydrogel group also exhibited significant difference $(P<0.01)$, indicating that the hydrogel possessed good hemostatic capacity in vivo. 
a)

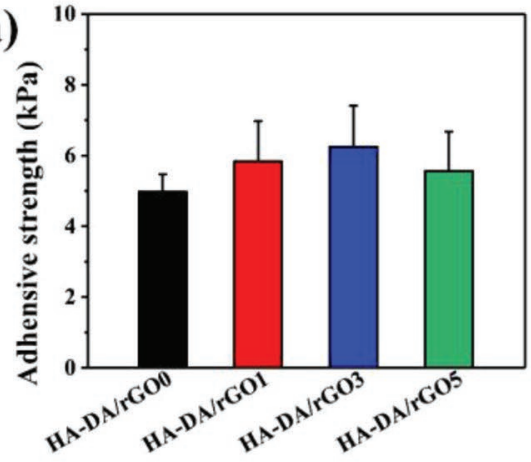

d)

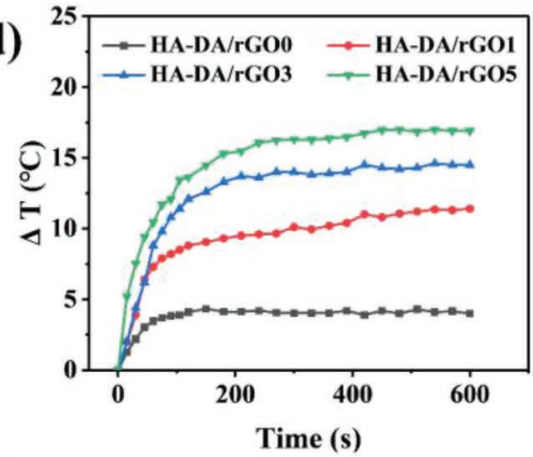

b)

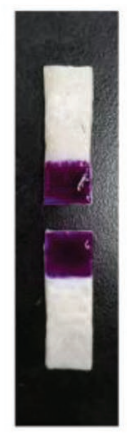

e)

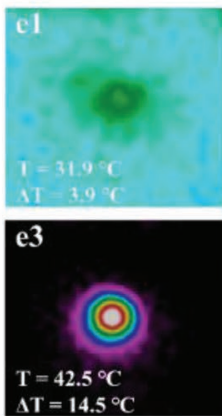

h)
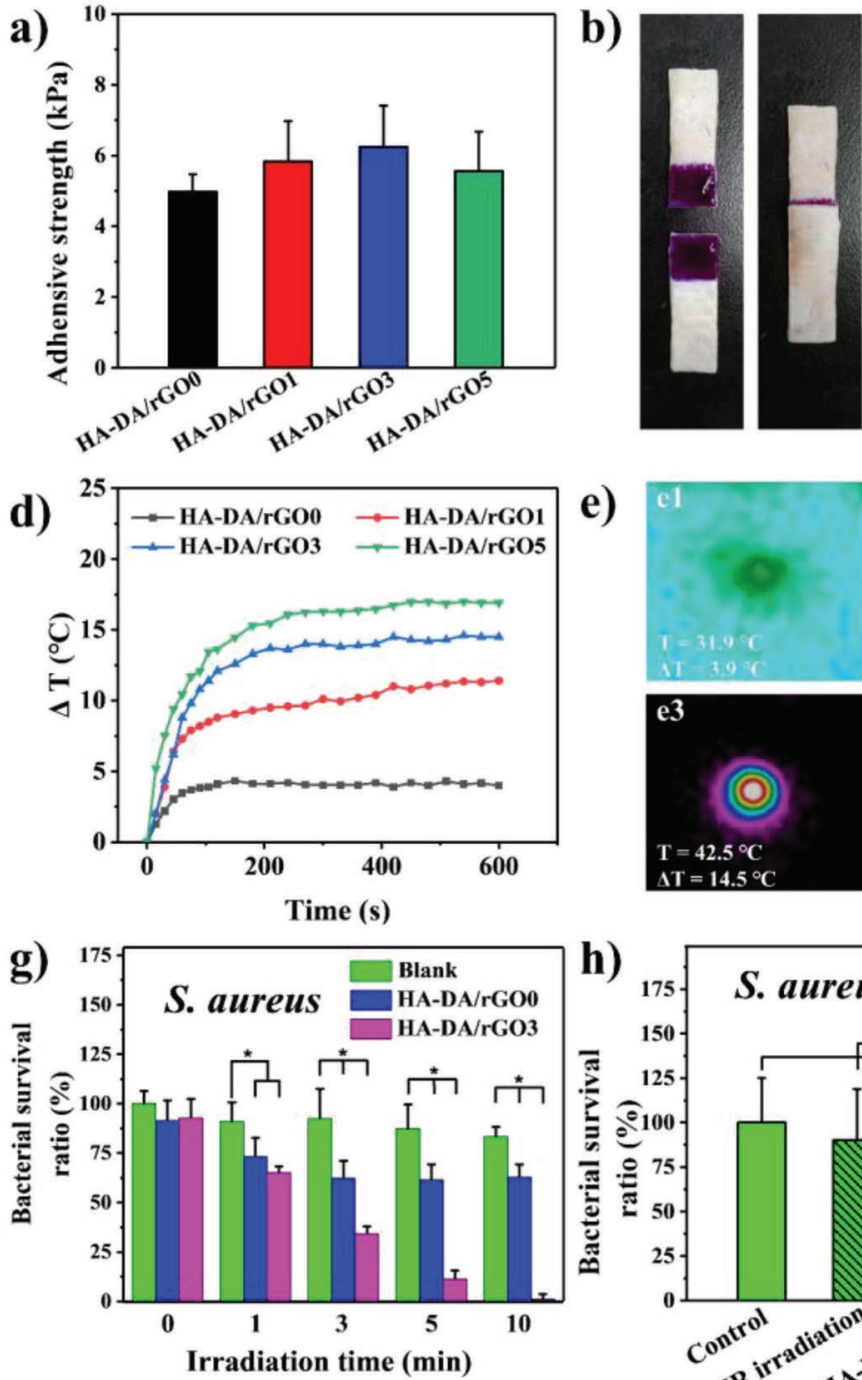

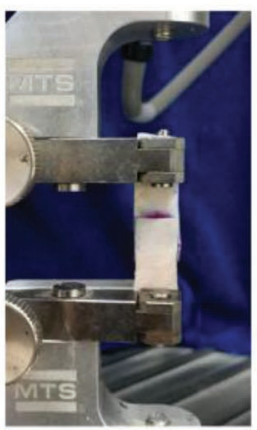

c)
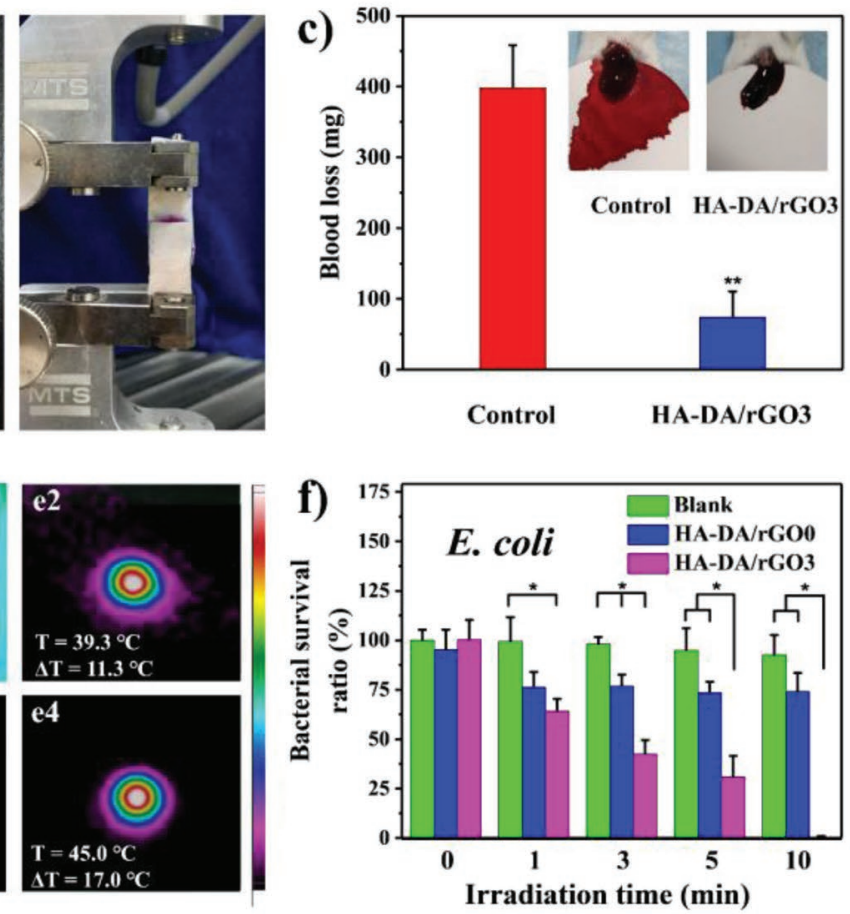

i)
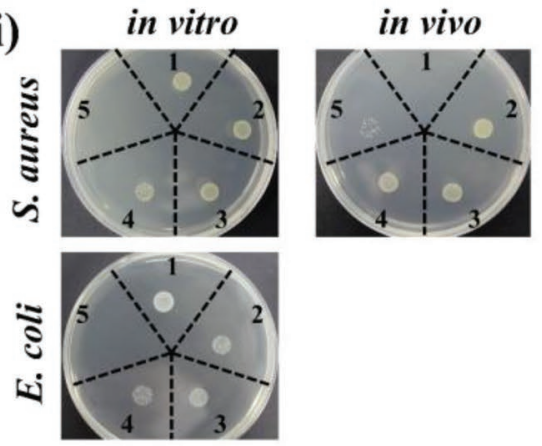

Figure 4. a) Adhesive strength of different hydrogels; b) Photographs of adhesive strength test; c) Hemostatic performance of hydrogel HA-DA/rGO3; d) The curves of $\Delta T$-NIR irradiation time for the hydrogels under light intensity of $1.0 \mathrm{~W} \mathrm{~cm}^{-2}$; Heat maps of el) HA-DA/rGO0, e2) HA-DA/rGO1, e3) HA-DA/rGO3, and e4) HA-DA/rCO5 hydrogels after 10 min NIR $808 \mathrm{~nm}$ irradiation; f) NIR irradiation enhanced in vitro antibacterial performance of hydrogels for E. coli and g) S. aureus; h) NIR irradiation enhanced in vivo antibacterial performance of hydrogels for S. aureus; i) Photographs of in vitro and in vivo antibacterial performance of hydrogels, and for the in vitro test, $1-5$ represented the different irradiation times from 0 to $1,3,5$, and 10 min, respectively. For the in vivo test, 1-5 represented the blank (PBS), PBS control, PBS+NIR irradiation, HA-DA/rGO3, and HA-DA/rGO3+NIR groups, respectively.

\subsection{Photothermal Property and Photothermal Antibacterial Activity}

The photothermal property of these hydrogels was estimated. The curves of temperature rise and NIR irradiation time for the hydrogels were recorded and shown in Figure 4d. HA-DA/ rGO0 showed the lowest temperature increase about $3.9^{\circ} \mathrm{C}$ after 10 min NIR irradiation due to the crosslinked catechol groups from HA-DA. With the rise of rGO@PDA mass ratio from 1 to 3 and $5 \mathrm{wt} \%$, the $\Delta T \mathrm{~s}$ increased from 11.3 to 14.5 and $17{ }^{\circ} \mathrm{C}$, indicating the good photothermal ability of rGO contained HA-DA/rGO hydrogels. Furthermore, Figure 4e exhibits the temperature pictures of these hydrogels after $10 \mathrm{~min}$ NIR irradiation, the core area with maximum temperatures from 32 to $45^{\circ} \mathrm{C}$ (corresponding $\Delta T$ from 3.9 to $17^{\circ} \mathrm{C}$ ) were surrounded by zones with a large temperature gradient.

Studies have shown that bacteria could be killed due to the damage of some enzymes and proteins when the temperature is above $50^{\circ}$. The NIR-induced in vitro photothermal antibacterial ability of HA-DA/rGO3 $\left(\Delta T\right.$ of $\left.14.5^{\circ} \mathrm{C}\right)$ was evaluated and HA-DA/rGO0 hydrogel and PBS were taken as the control groups (Figure 4f,g). When introducing rGO@PDA as photothermal contrast agent, for just $1 \mathrm{~min}$ of NIR irradiation, HA-DA/rGO3 showed significantly decreased $(P<0.05)$ bacteria survival ratio of $64.1 \%$ for Escherichia coli and $65.4 \%$ for Staphylococcus aureus. Moreover, with the time of irradiation increased, the survival ratio of bacteria showed a cliff-like decline. When the irradiation time increased to $10 \mathrm{~min}$, 
HA-DA/rGO3 group showed nearly complete bacteria killing for both the two bacteria. For HA-DA/rGO0 group, after $10 \mathrm{~min}$ irradiation, the bacterial survival ratios just decreased to $74.1 \%$ and $62.9 \%$ for E. coli and S. aureus. These results demonstrated that the HA-DA/rGO hydrogel possessed excellent in vitro NIR photo-thermal performance, which are beneficial to a good antibacterial ability for both gram-negative and gram-positive bacteria.

To further confirm the photothermal antibacterial effect of hydrogels in vivo, a $S$. aureus infected skin wound model of mouse was established. After 2 days' infection with $S$. aureus, all the wounds became inflamed and had a light yellow exudate with the amount of bacterial colonies reaching $10^{8} \mathrm{CFU}$ from the original $10^{6} \mathrm{CFU}$, indicating the successful establishment of infection model. As shown in Figure $4 \mathrm{~h}$, after different treatment and culturing bacteria from the infected tissues, LB agar plates from treated tissues groups suggested that the PBS control group and wound treated with PBS+NIR irradiation and HA-DA/rGO3 hydrogel exhibited high bacterial survival ratios, while the wound covered with HA-DA/rGO3 hydrogel and applied $10 \mathrm{~min}$ NIR irradiation showed a very low bacterial survival ratio (3.1\%), and the HA-DA/rGO3+NIR group and the other three groups also showed significant difference $(P<0.01)$. Besides, the bacterial survival ratios among PBS control, PBS+NIR irradiation and HA-DA/rGO3 hydrogel groups showed no obvious difference, indicating that the antibacterial property of HA-DA/rGO originated from the photo-thermal effect provided by rGO. Besides, the in vivo antibacterial activity of HA-DA/rGO hydrogels against E. coli was also carried out (Figure S6, Supporting Information). A very low E. coli survival ratio $(3.1 \%)$ was observed when the wound was covered with HA-DA/rGO3 hydrogel and applied $10 \mathrm{~min}$ NIR irradiation, demonstrating the broad-spectrum photothermal antimicrobial activity of the HA-DA/rGO hydrogels.

The macroscopic photos (Figure $4 \mathrm{i}$ and Figure S7, Supporting Information) of in vitro antibacterial performance from different irradiation times $(0,1,3,5$, and $10 \mathrm{~min})$ for $S$. aureus and E. coli and in vivo antibacterial ability from different treatments for both of them also agreed well with the antibacterial effect in Figure $4 \mathrm{f}-\mathrm{h}$. These results confirmed the good in vivo and in vitro photothermal antibacterial ability of $\mathrm{HA}-\mathrm{DA} / \mathrm{rGO}$ hydrogel.

\subsection{Drug Release and Antibacterial Activity of the Hydrogel}

As widely used broad-spectrum antimicrobial agents, the clinical application of tetracycline ointment has strongly confirmed the application of tetracyclines drugs in skin wound repair. ${ }^{[58]}$ Hence, doxycycline as a well-known "second generation" tetracycline in clinical was used to endow the hydrogel with antibacterial activity, and the release profiles of it from the hydrogels was studied. As can be seen in Figure 5a, doxycycline' release behavior within the first $15 \mathrm{~h}$ showed a nearly linear release and had no apparent difference among the four groups. After that, the release speed decreased, but the release process lasted for about $10 \mathrm{~d}$ and $\approx 77.5 \%, 80 \%, 83.8 \%$, and $87 \%$ of doxycycline were released in PBS at $\mathrm{pH} 7.4$ from HA-DA/rGO5, HA-DA/rGO3, HA-DA/rGO1 and HA-DA/rGO0, respectively.
These results demonstrated the sustained release behavior of doxycycline from HA-DA/rGO hydrogel and the potential of the HA-DA/rGO hydrogel used as carrier for sustained drug release. FT-IR test was employed to characterize the interactions of doxycycline to HA-DA/rGO hydrogel (Figure S8, Supporting Information), there were some characteristic peaks at 3451 and $3332 \mathrm{~cm}^{-1}$ representing amino group in the FT-IR spectrum of doxycycline. However, these peaks disappeared and shifted to a lower wavenumber in the FT-IR spectrum of HA-DA/rGO/ Doxy hydrogel, indicating the existence of hydrogen bond between amino group of doxycycline and hydrogel network, which greatly prolonged the sustained release time of the drug. As to the further mechanism of doxycycline sustained release from the hydrogels, the famous Korsmeyer-Peppas equation was used and the results exhibited that the cumulative release of doxycycline can be well fitted by this equation with high correlation $\left(r^{2}>0.99\right)$

$M \mathrm{t} / M_{\infty}=k * t^{n}$

In particular, $n$ represents the diffusion exponent in this equation. And for cylindrical samples, the release behavior could be described by Fickian diffusion if $n$ is less than 0.45 . The fitted data (Figure 5b) exhibited that the $n$ values for all of the hydrogels were between 0.31 and 0.33 , indicating that the doxycycline was released from the HA-DA/rGO hydrogels by diffusion.

In order to observe the antibacterial activity of doxycycline loaded HA-DA/rGO hydrogel with controlled release property, zone of inhibition (ZOI) test was performed on both the gramnegative bacterial E. coli and gram-positive bacterial $S$. aureus, respectively. Figure 5c,d shows the changes of dark gray zones (marked with a red circle) of bacterial inhibition versus time with different bacteria. As comparison, PBS and drugfree HA-DA/rGO3 hydrogel did not show any zone of clearance throughout the experiment. In contrast, the entire drug release process of doxycycline encapsulated HA-DA/rGO3 hydrogel lasted a long time of $9 \mathrm{~d}$ for $E$. coli and $15 \mathrm{~d}$ for $S$. aureus, respectively, due to the sustained release of doxycycline to the surroundings which inactivated bacteria in the respective areas (Figure 5c,d). The statistical data of inhibition zones also decreased from 3.9 to $1.7 \mathrm{~cm}$ for $S$. aureus and from 3.2 to $1.5 \mathrm{~cm}$ for $E$. coli, due to the gradually decreased doxycycline release content (Figure 5e).

\subsection{Biocompatibility Evaluation of the Hydrogels}

Good biocompatibility is an essential factor for biomaterials. In vitro hemolysis test was performed to estimate the hemocompatibility of HA-DA/rGO hydrogel. The picture in Figure $6 \mathrm{~b}$ exhibited the apparent difference in color between the four hydrogels group, negative control group (PBS) and positive control group (Triton X-100). All the four hydrogel groups were observed to be light yellow, which was similar to the negative control group, while the Triton X-100 group was bright red. The quantitative data are presented in Figure 6a, and HA-DA/rGO0 hydrogel exhibited a very low hemolysis ratio (1.2\%), indicating the best hemocompatibility among the four hydrogels. When 

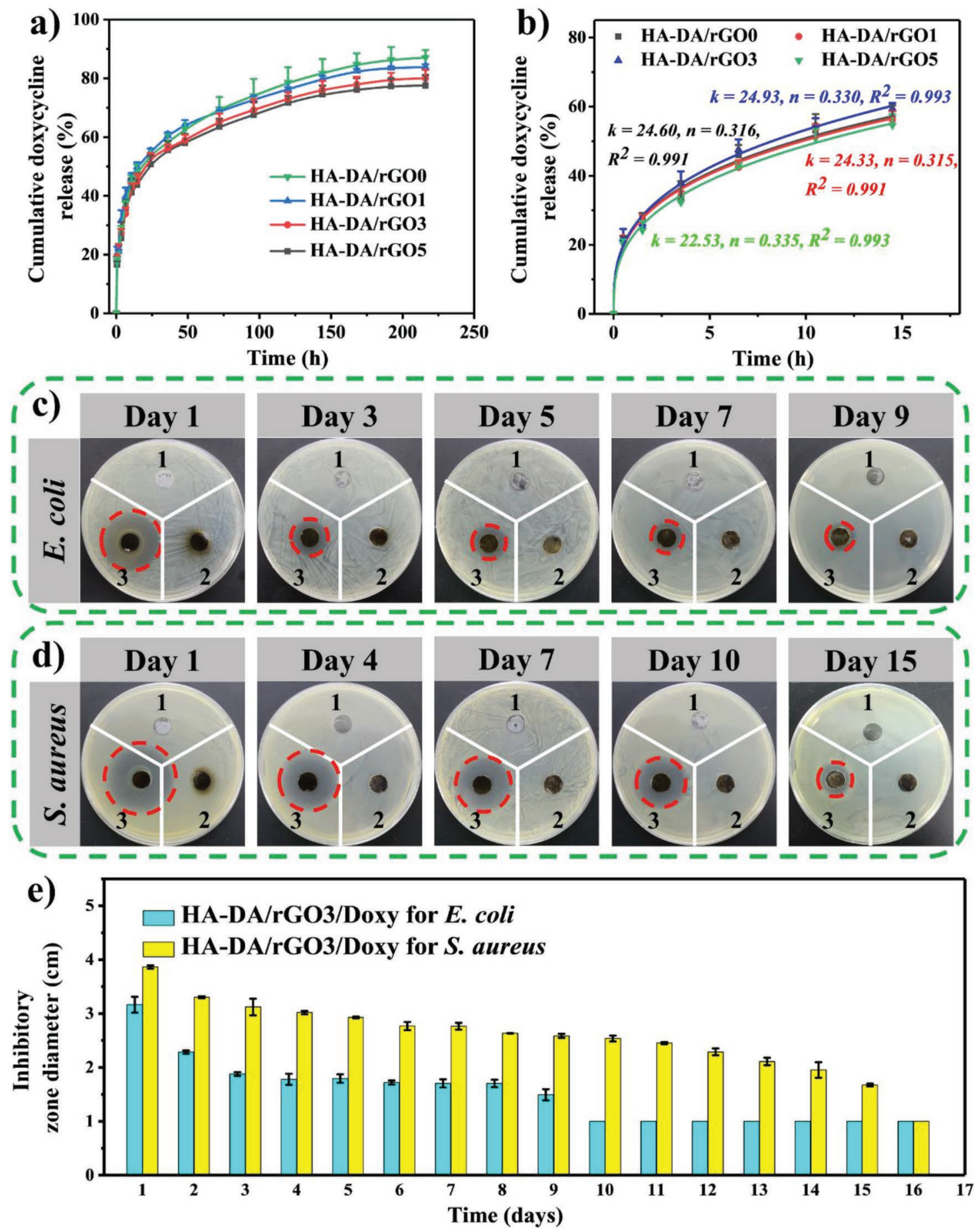

Figure 5. a) The in vitro cumulative release curves of doxycycline from hydrogels; b) The release profiles of doxycycline were fitted by Equation (1); Pictures of inhibition zones for bacterial from the sterilizing effectiveness test for c) E. coli and for d) S. aureus: (1) Blank, (2) HA-DA/rGO3 hydrogel, and (3) HA-DA/rGO3/Doxy hydrogel; e) Statistics of inhibitory zone diameter.

rGO@PDA was added, the hemolysis ratios showed no obvious increase (1.5\%-1.6\%).

To further evaluate the cytocompatibility of these hydrogels, a direct contact test between the L929 cells and hydrogels was carried out. As shown in Figure 6c, the number of cells on the fifth day increased significantly from the first day, demonstrating that the cells grew well throughout the whole experiment. Cell viability of all the groups on the first day showed no obvious difference demonstrated the nontoxicity of HA-DA/rGO hydrogel. With three days' coincubation, HA-DA/rGO1 and HA-DA/rGO3 hydrogels exhibited higher cell number than the TCP group $(P<0.05)$, and HA-DA/rGO3 hydrogel showed 
a)
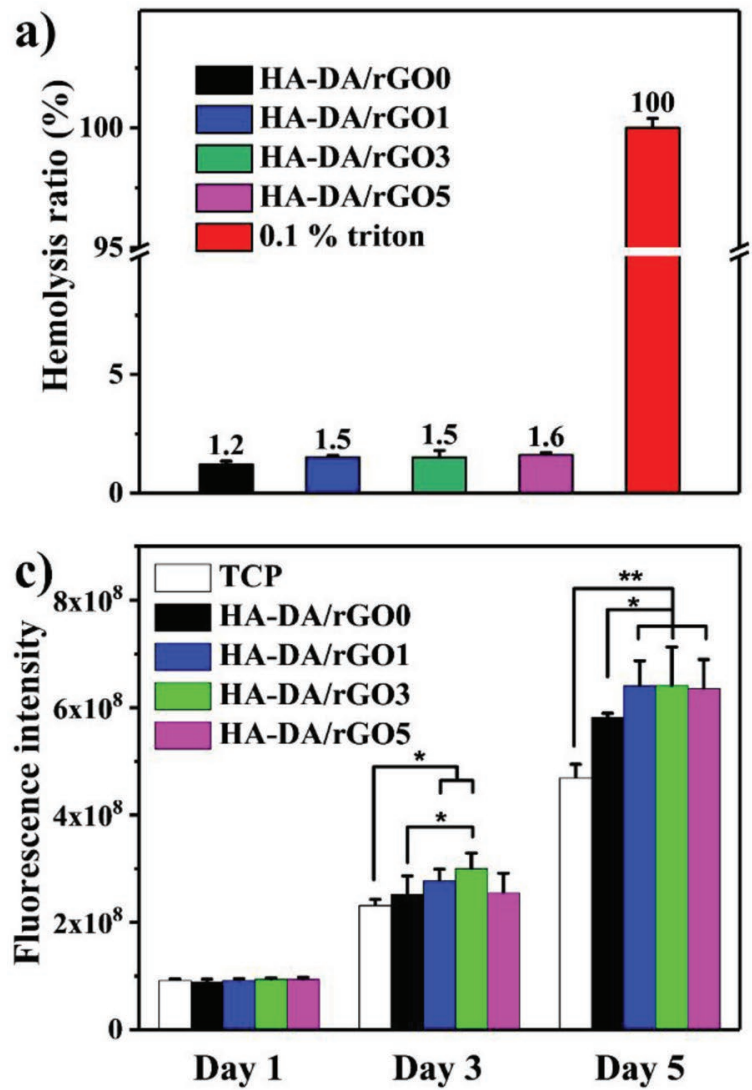

b)

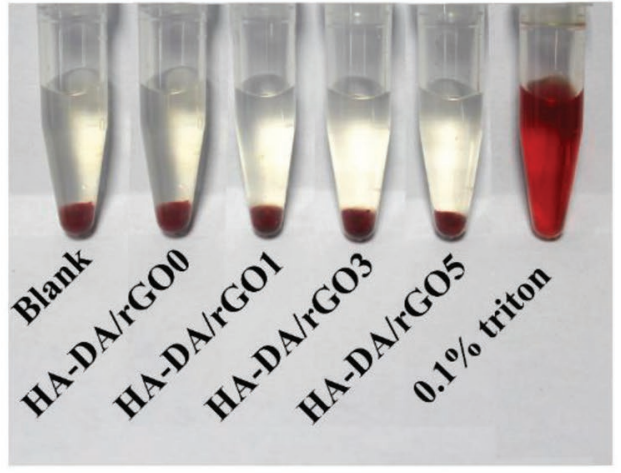

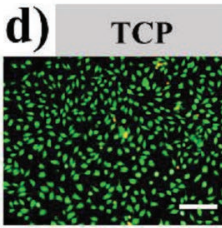
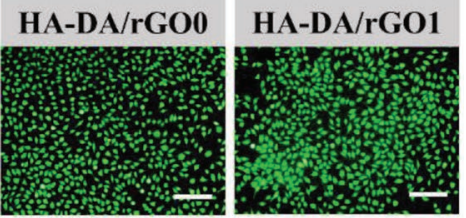

HA-DA/rGO3
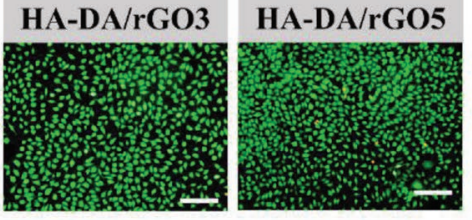

Figure 6. a) Hemolytic percentage of the hydrogels; b) Pictures from hemolytic activity test of the hydrogels; c) Cytocompatibility evaluation of these hydrogels by contacting with the L929 cells, $* P<0.05$, $* * P<0.01$; d) LIVE/DEAD staining of L929 cells after contacted with the hydrogels for 5 d. Scale bar: $200 \mu \mathrm{m}$

the highest cell proliferation. After five days' coincubation, all the hydrogel groups presented higher fluorescence intensity compared with TCP $(P<0.05)$, and HA-DA/rGO1, HA-DA/ rGO3 and HA-DA/rGO5 hydrogels exhibited significant difference $(P<0.05)$ with HA-DA/rGO0 hydrogel, indicating the promoting effect of rGO@PDA on cell proliferation. The pictures in Figure $6 \mathrm{~d}$ were also consistent with the quantitative experiments. The above experiments confirmed the good biocompatibility and wide application prospects of these hydrogels as hemostatic and wound healing materials. The HA-DA/ rGO3 with balanced rGO@PDA content showing best cell proliferation was selected for further evaluation of in vivo wound healing.

\subsection{In Vivo Wound Healing in a Full-Thickness Skin Defect Model}

To further evaluate the wound healing application, a mouse full-thickness skin defect repair test was performed to confirm the advantages of HA-DA/rGO hydrogel as wound dressing in vivo. HA-DA/rGO3 hydrogel with the best promoting effect for cell proliferation was chosen as a representative, and a Tegaderm dressing was used as a control group. Doxycycline-loaded hydrogel (HA-DA/rGO3/Doxy) was used as another group because of its good antibacterial ability (Figure $5 \mathrm{e}$ ). As can be seen in Figure $7 \mathrm{a}-\mathrm{c}$, the wound areas in all the four groups became smaller with increasing postsurgery time. After treated for $3 \mathrm{~d}$, the wound areas treated with HA-DA/rGO0, HA-DA/ rGO3 and HA-DA/rGO3/Doxy hydrogels were smaller than Tegaderm dressing $(P<0.05)$. HA-DA/rGO3 group and HA-DA/ rGO3/Doxy group also showed significant difference $(P<0.05)$ with HA-DA/rGO0 hydrogel. However, there was no significant difference between HA-DA/rGO3 and HA-DA/rGO3/Doxy groups $(P>0.05)$. After treated for $7 \mathrm{~d}$, all of the three hydrogel groups still showed significantly enhanced wound repair rate than Tegaderm dressing group $(P<0.05)$. On the 14th day, the wounds treated with Tegaderm dressing had passably closed, but it showed completely closed in the hydrogel group, and even some of the mice presented hair coverage. Quantitative analysis of the wound area over the whole repair process indicated that the wounds covered with HA-DA/rGO3 and HA-DA/ rGO3/Doxy hydrogels had healing ratios of $95.5 \%$ and $98.0 \%$ (Figure 7c), which exhibited significant difference with the Tegaderm dressing $(90.2 \%)(P<0.05)$ and HA-DA/rGO0 group (92.8\%) $(P<0.05)$, indicating the better effect of HA-DA/rGO3 and HA-DA/rGO3/Doxy hydrogel group on accelerating wound healing. Besides, the healing rate of HA-DA/rGO3 hydrogel exhibited no obvious difference with the HA-DA/rGO3/Doxy hydrogel $(P>0.05)$, indicating that the HA-DA/rGO3 hydrogel was intrinsically beneficial to wound healing. All these in vivo evaluation results demonstrated that the HA-DA/rGO hydrogel 

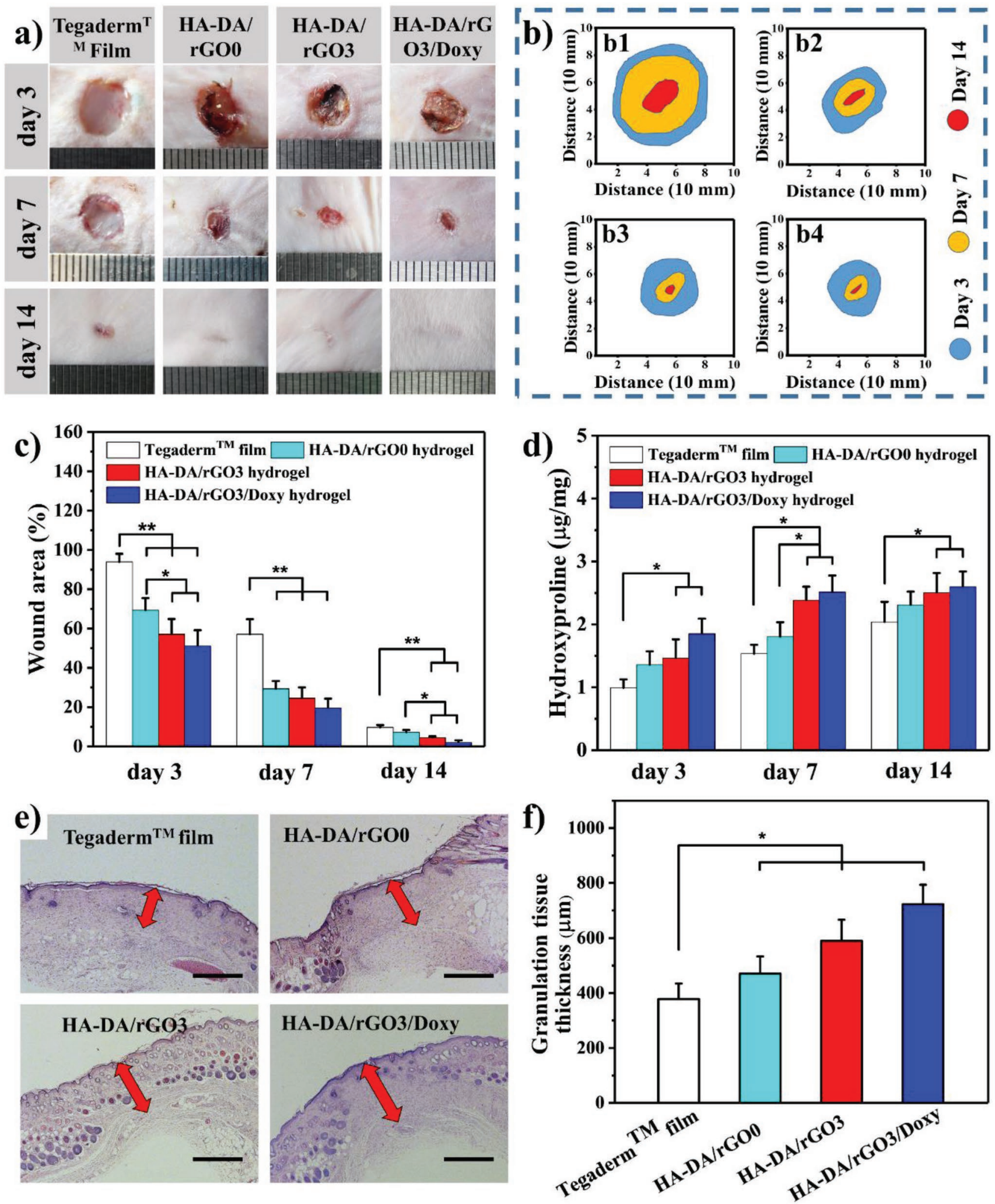

Figure 7. a) Photographs of wounds on $3 \mathrm{rd}$, 7th, and 14 th day for Tegaderm film dressing (control), $\mathrm{HA}-\mathrm{DA} / \mathrm{rGOO}, \mathrm{HA}-\mathrm{DA} / \mathrm{rGO}$, and $\mathrm{HA}-\mathrm{DA} / \mathrm{rGO} 3 /$ Doxy hydrogel; b) Schematic diagram of wound area during $14 \mathrm{~d}$ for b1) Tegaderm film dressing, b2) HA-DA/rGO0, b3) HA-DA/rGO3, and b4) HA-DA/ rGO3/Doxy; c) Wound area for each group. $* P<0.05$, $* * P<0.01$; d) Collagen amount in different groups by measuring the content of hydroxyproline. $* P<0.05$; e) Granulation tissue (red arrows) thickness for different groups on 14th day. Scale bar: $500 \mu m$. f) Quantitative data of granulation tissue thickness. 
with adhesive hemostatic property showed great promoting effect in wound-healing compared with Tegaderm dressing.

Throughout the whole healing process, metabolism of collagen is constantly changing. Hydroxyproline is unique in collagen fibers, and its mass fraction is constant (13.4\%) in collagen. ${ }^{[59]}$ Therefore, the hydroxyproline content was measured to evaluate the collagen deposition in the new connective tissue. As can be seen in Figure $7 d$, collagen content in each group continued to rise throughout the $14 \mathrm{~d}$ period. Besides, compared with control group, all of the hydrogel groups showed higher collagen over the whole repair stage, and the HA-DA/rGO3 and HA-DA/rGO3/Doxy groups exhibited obvious difference with the control group $(p<0.05)$ in the whole test period. Especially, HA-DA/rGO3 and HA-DA/rGO3/ Doxy groups even exhibited significant difference when compared with the HA-DA/rGO group $(p<0.05)$ on the seventh day, and HA-DA/rGO3 hydrogel still had no obvious difference with the HA-DA/rGO3/Doxy hydrogel $(P>0.05)$, indicating that hydrogel HA-DA/rGO3 greatly enhanced the collagen deposition compared to HA-DA/rGO0 hydrogel.

For the injury accompanied with inflammation, it is difficult for the parenchymal cells to complete the repair work alone. ${ }^{[60]}$ In these circumstances, granulation tissue plays a vital role in the repair process. It will first proliferate, and then dissolve and absorb necrotic tissue and foreign bodies, filling the gap and finally transformed into scar tissue to complete the repair. ${ }^{[61]}$ Hence, a thicker granulation tissue in the wound healing process is an important indicator to evaluate the repair effects. As shown in Figure 7e-f, after 14 days' repair, the granulation tissue in Tegaderm films group was thinner than all of the hydrogel groups $(P<0.05)$. Among the hydrogel groups, HA-DA/rGO3 hydrogel produced a thicker granulation tissue than HA-AD/rGO0 hydrogel, and HA-DA/rGO3/Doxy showed the thickest granulation tissue, indicating the best wound healing effect in these three groups.

\subsection{Histomorphological Evaluation}

To further distinguish the quality of regenerated skin in the defects among the four groups, histological analysis was performed (Figure 8a). ${ }^{[62]}$ By monitoring the wounds in the early stages of wound healing (3-7 d), no obvious inflammatory response which triggered by these hydrogels was observed for all the groups, although more inflammatory cells were found in wound sites of Tegaderm film group than the three hydrogel groups (Figure 8a-a1-a4) through the hematoxylin eosin (H\&E) staining on the third day. These results demonstrated that the HA-DA/rGO hydrogels did not cause increased foreign body response. With the advancement of the repair process, inflammatory cells were less observed for all the groups on the seventh day. Additionally, the three experimental groups showed different degrees of nearly complete and thicker epidermis compared with the blank group on the seventh day, especially the HA-DA/rGO3 and HA-DA/rGO3/Doxy group (Figure 8b). In general, new blood vessels are essential to wound repair by providing nutrients and oxygen to the damaged tissue microenvironment. As shown in Figure 8d, HA-DA/rGO3 and HA-DA/ rGO3/Doxy hydrogel groups exhibited more blood vessels than control group $(P<0.05)$, and even some hair follicles in HA-DA/rGO3/Doxy group formed (Figure 8a-b4). On the 14th day, although Tegaderm films group exhibited complete re-epithelialization, few hair follicles were observed (Figure 8a-c1,c). In contrast, the wound treated with the hydrogels, especially HA-DA/rGO3 and HA-DA/rGO3/Doxy hydrogels, was covered with epithelial tissue that is closer to normal skin (Figure 8b). ${ }^{[6]}$ More skin appendages like hair follicles (Figure 8c) and blood vessels (Figure 8d) were observed in HA-DA/rGO3 and HA-DA/ rGO3/Doxy hydrogel groups, and also exhibited significant differences when compared with control and HA-DA/rGO0 groups $(P<0.05)$, respectively. These results indicating that the hydrogel, especially those with rGO were beneficial to ECM remodeling and tissue regeneration. Graphene with high specific surface area exhibits favorable interaction pattern with tissues, cells and even biomolecules, thus improving the rGO incorporated hydrogel's bioactivity to enhance the wound healing process. ${ }^{[36,37]}$ In addition, the unique structure of graphene makes it easy for electrons to escape from graphene into cells under the action of the cell membrane. ${ }^{[64]}$ The conductivity of rGO might contribute to the skin tissue regeneration via transmitting electrical signals between wound sites and stimulating excitable skin cells.

\subsection{TNF- $\alpha$ and CD31 Expression During the Wound Healing Process}

Numerous studies have shown that cytokines are specifically associated with the initiation and cessation of many required cellular activities for wound healing. ${ }^{[65,66]}$ Tumor necrosis factor- $\alpha$ (TNF- $\alpha$ ) was selected to estimate the effect of these hydrogel dressings in preventing infection. In Figure 9a,c, the antibiotic doxycycline loaded HA-DA/rGO3/Doxy hydrogel exhibited lower expression of TNF- $\alpha$ compared with other groups on both 7 th day and 14 th day $(p<0.05)$. The HA-DA/ rGO0 and HA-DA/rGO3 groups also exhibited significant differences with Tegaderm films group $(p<0.05)$, confirming the better effects of these hydrogels than control group. Angiogenesis of the wound was determined by CD31 immunohistochemical staining of healed wounds at day 7 and day 14 postsurgery in this study. As can be seen in Figure 9b,d, the wounds treated with HA-DA/rGO3 and HA-DA/rGO3/Doxy groups appeared grossly more CD31 than Tegaderm films group on 7th and 14th day $(P<0.05)$, illustrating the advantages of the addition of conductive component rGO@PDA and antibiotics doxycycline in accelerating wound closure and improving angiogenesis. Besides, there is no difference between the HA-DA/rGO3 and HA-DA/rGO3/Doxy group in the angiogenesis. In summary, by reducing the expression of proinflammatory factor (TNF- $\alpha$ ) and upregulating production of CD31 simultaneously, hydrogel groups, especially HA-DA/rGO3 and HA-DA/rGO3/Doxy groups, significantly promoted wound closure and induced a better repair efficiency than the Tegaderm films.

In conclusion, HA-DA/rGO0 hydrogel showed better repair effect than the Tegaderm film group, mainly due to the promotion effect of hyaluronic acid for wound healing. Besides, the HA-DA/rGO3 hydrogel containing rGO@PDA exhibited better skin tissue regeneration and ECM remodeling than the HA-DA/ rGO0 hydrogel in terms of wound closure rate, granulation 

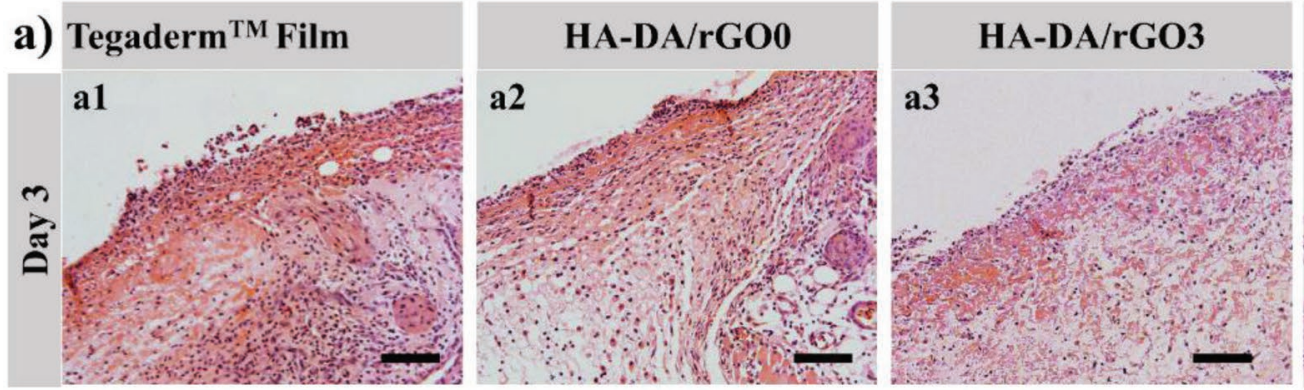

\section{HA-DA/rGO3/Doxy}
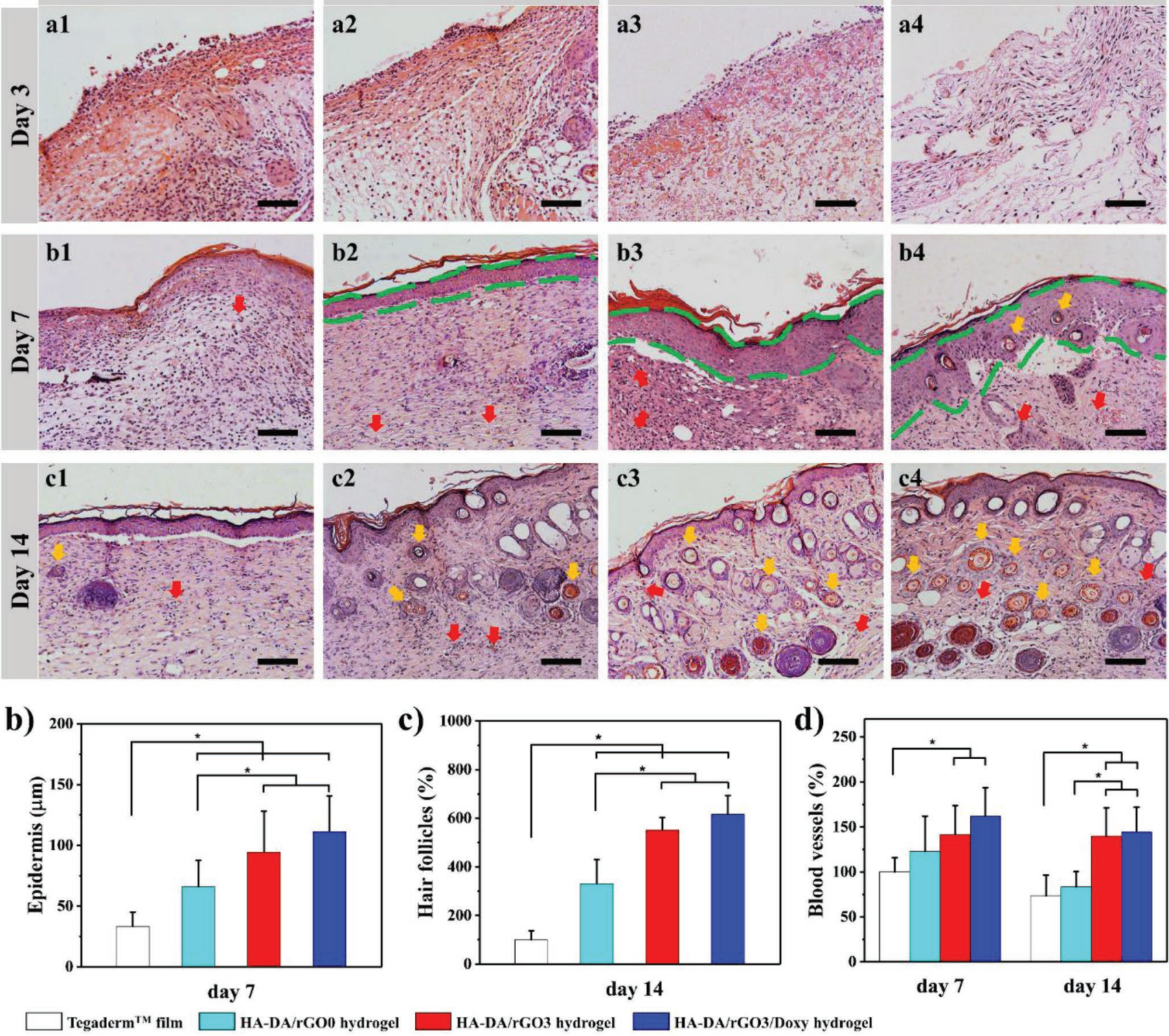

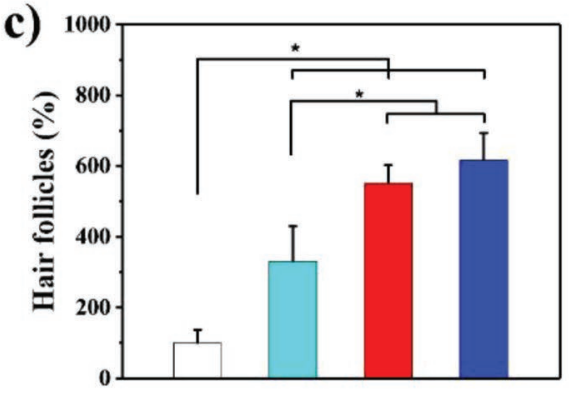

day 14

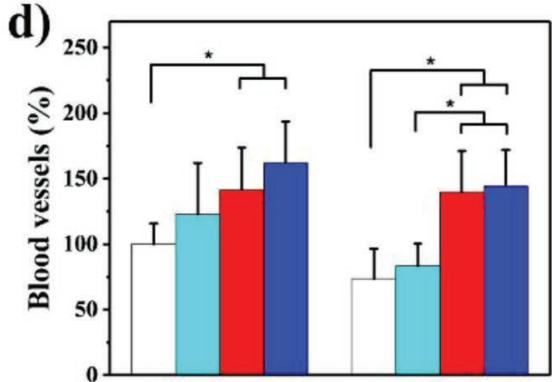

day 7

day 14

Figure 8. a) Pictures of histological analysis for wound regeneration among Tegaderm film dressing, HA-DA/rGOO, HA-DA/rGO3, and HA-DA/rGO3/ Doxy hydrogels on 3rd, 7th, and 14th day (blood vessels: red arrows, hair follicles: yellow arrows, boundary of epithelium: green lines). Scale bar: 100 um; b) Epidermis thickness for different groups on 7th day; c) Hair follicles for different groups on 14th day; d) Blood vessels regeneration on 7th and 14th day.

tissue thickness, collagen deposition, epidermis, hair follicles, and vessel blood regeneration, indicating the incorporation of rGO@PDA greatly improved wound healing effect of hydrogel. As additional functions, adhesive and mechanical properties are presented in all three hydrogel groups, providing good hemostasis, tissue closure, and robust physical barrier to accelerate wound closure.

\section{Conclusion}

A series of adhesive hemostatic antioxidative and conductive hydrogels based on hyaluronic acid-graft-dopamine and polydopamine-coated reduced graphene oxide were successfully prepared through the crosslinking of catechol groups with $\mathrm{HRP} / \mathrm{H}_{2} \mathrm{O}_{2}$ as initiator system. These hydrogels exhibited multifunctions like tunable gelation time, stable rheological property, suitable swelling, and degradation behavior, excellent biocompatibility, inherent photo-thermal effect, NIR irradiation induced and doxycycline endowed antibacterial activity. Besides, more than 10 days' drug release study in physiological environment and zone of inhibition test of antibiotics loaded hydrogels exhibited the promising application of these hydrogels in drug sustained release carriers. Additionally, good tissue adhesion, excellent stretching, compression, and bending property also make these hydrogels more competitive in the application of 

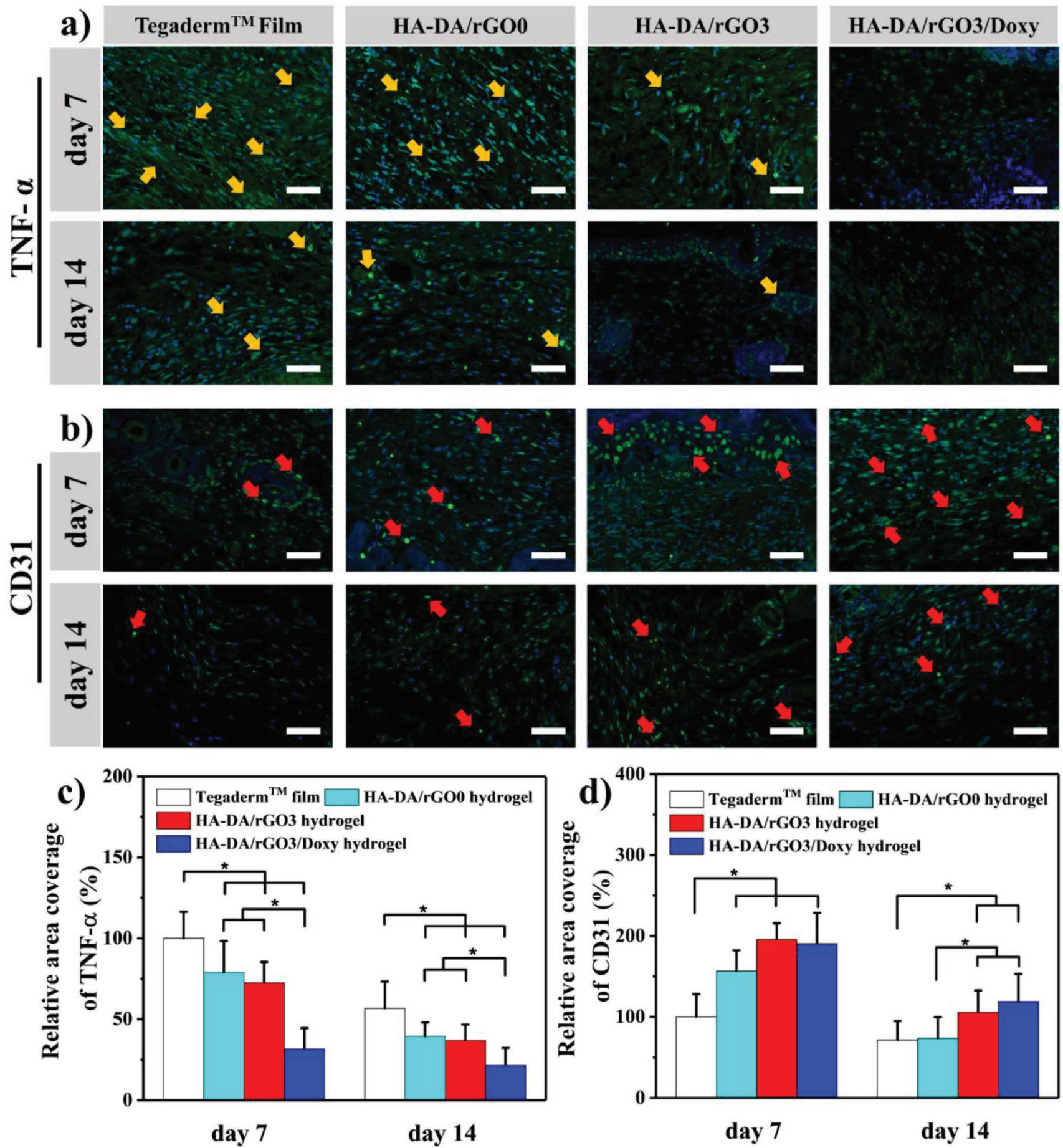

Figure 9. Pictures of skin wound tissues on 3rd day, 7th day, and 14th day after surgery by immunofluorescence labeling with a) TNF- $\alpha$ (green) and b) CD31 (green), yellow arrows show the hair follicles and red arrows present the blood vessels. Scale bar: $100 \mu \mathrm{m}$. Quantified data of the relative area percentage that covered by c) TNF- $\alpha$-actinin and d) CD31, respectively. For all data, the control group on seventh day was set as $100 \%$. $* P<0.05$.

wound dressings. An antioxidant property and excellent hemostatic effects were also demonstrated by a free radical scavenging test and mouse liver injury model. Furthermore, the rGO@PDA added hydrogel HA-DA/rGO3 showed better in vivo wound healing effect than commercial Tegaderm film and rGO/ PDA absent HA-DA/rGO hydrogel in terms of wound closure, granulation thickness, and collagen metabolism. Especially, histomorphological evaluation and TNF- $\alpha$ and CD31 expressions during the wound healing process proved the better promoting effects of these multifunctional hydrogels in process of wound healing in a full-thickness mouse skin wound model. All these results demonstrated that adhesive conductive hemostatic 
hydrogel with multiple functions are ideal candidates as drug sustained release carriers and wound healing dressings.

\section{Experimental Section}

Synthesis of Hyaluronic Acid-Graft-Dopamine: The hyaluronic acidgraft-dopamine conjugate was prepared by an EDC chemistry procedure following previous studies with some modifications. ${ }^{[27,67]}$ The specific synthesis procedures are available in Supporting Information.

Synthesis of Polydopamine-Coated CO (rGO@PDA): rGO@PDA was prepared by stirring $\mathrm{GO}$ and dopamine hydrochloride together in an alkaline condition (Tris- $\mathrm{HCl}$ buffer, $\mathrm{pH}=8.5$ ). ${ }^{[68,69]}$ And the details are available in Supporting Information.

Preparation of HA-DA/rGO Hydrogel: To prepare the HA-DA/rGO hydrogel, ${ }^{, 70]} \mathrm{HA}-\mathrm{DA}$ aqueous solution and rGO@PDA aqueous dispersion were cross-linked through an oxidative coupling by using $\mathrm{H}_{2} \mathrm{O}_{2} / \mathrm{HRP}$ as initiator system. First, HA-DA was added to PBS to form a $2.5 \mathrm{wt} \%$ solution at $37^{\circ} \mathrm{C}$. rGO@PDA was dispersed in PBS varying the rGO@PDA from 0.2 to 0.6 and $1 \mathrm{wt} \%$, respectively at room temperature. $1 \mathrm{~mL}$ of above rGO@PDA dispersion or $1 \mathrm{~mL}$ of DI water was added into $8 \mathrm{~mL}$ of HA-DA solution to prepare the mixed solutions. Following completely stirred, $0.5 \mathrm{~mL}$ of $\mathrm{H}_{2} \mathrm{O}_{2}$ (Sigma-Aldrich) solution $\left(0.1 \mathrm{~mol} \mathrm{~mL}^{-1}\right)$ and $0.5 \mathrm{~mL}$ of HRP (Sigma-Aldrich) solution $\left(0.5 \mathrm{mg} \mathrm{mL}^{-1}\right)$ were added to HA-DA/ rGO mixture. Then, the hydrogel precursor was placed at $37^{\circ} \mathrm{C}$ and the hydrogel can be formed after a short time. Hydrogels with a 2 wt\% HA-DA content and increasing weight ratios of rGO@PDA to HA-DA varying from $0,1,3$, and $5 \mathrm{wt} \%$ were prepared in this study.

Characterizations: The FT-IR, nuclear magnetic resonance ('H NMR), UV-vis spectroscopy, SEM, and rheological measurements were used to confirm the physical and chemical characterizations of HA-DA, rGO@PDA, or HA-DA/rGO hydrogels. The specific details of thermal stability, ${ }^{\left[{ }^{[1]}\right.}$ conductivity test, ${ }^{[72]}$ swelling, ${ }^{[9,73]}$ and degradation tes ${ }^{[74]}$ are available in the Materials and methods section of Supporting Information.

Rheological Measurements of the Hydrogels: The TA rheometer (DHR-2) was used to test the rheological property of these hydrogels. The stiffness of these hydrogels was measured by a time sweep test with a constant strain of $1 \%$ and frequency of $10 \mathrm{rad} \mathrm{s}^{-1}$ at $37^{\circ} \mathrm{C} .350 \mu \mathrm{L}$ of the polymer mixture was placed at a height of $1000 \mu \mathrm{m}$ between $20 \mathrm{~mm}$ parallel plates and sealed with silicone oil to prevent water evaporation. ${ }^{[75]}$

Mechanical Properties of HA-DA/rGO Hydrogel: Compression and tensile stress-strain evaluation of HA-DA/rCO hydrogel were performed based on our previous reports. ${ }^{[73,76]}$ The details are available in the Materials and methods section of Supporting Information.

Antioxidant Ability of Hydrogels: The efficiency of scavenging the stable 1,1-diphenyl-2-picrylhydrazyl (DPPH) free radical was used to evaluate the antioxidant ability of these hydrogels by referring to previous reports. ${ }^{[77]}$ The details are available in the Materials and methods section of Supporting Information.

Adhesive Strength Test of the Hydrogels: The adhesive strength test of hydrogel was conducted based on our previous reports. ${ }^{[55,78]}$ The details of the test are available in the Materials and methods section of Supporting Information.

Hemostatic Ability Test In Vivo: According to the ref. [12], a liver bleeding mouse model (Kunming mice, 20-30 g, female) was established to evaluate the hemostatic effect of these HA-DA/rGO hydrogels. The details of specific operation steps are available in the Materials and methods section of Supporting Information. All measurements were carried out more than five times. All animal experiments were conducted based on current guidelines on the care of experimental animals and approved by professional committee of Xi'an Jiaotong University.

Photothermal Property of the Hydrogels: For the evaluation of photothermal property of these hydrogels, the as-prepared hydrogel was cut into cubes $(5 \times 5 \times 5 \mathrm{~mm})$ and then exposed to an NIR laser (MDL-III-808 nm-1000 mW, Changchun New Industries Optoelectronics Tech Co., Ltd.) with a power density of $1.0 \mathrm{~W} \mathrm{~cm} \mathrm{~cm}^{-2}$ for $10 \mathrm{~min}$. The temperature pictures of these hydrogels were recorded using an infrared (IR) thermal camera. ${ }^{[79]}$
NIR Irradiation Enhanced In Vitro and In Vivo Antibacterial Performance of Hydrogels: The test of NIR irradiation enhanced in vitro antibacterial performance was carried out by a modified method from our previous work. ${ }^{[76]}$ The hydrogel was exposed to NIR laser light $\left(808 \mathrm{~nm}, 1.0 \mathrm{~W} \mathrm{~cm}^{-2}\right)$ for different time from 0 to $1,3,5$, and $10 \mathrm{~min}$, respectively. ${ }^{[80]}$ The details are available in the Materials and methods section of Supporting Information. At least three samples in each group were tested and the results were expressed as bacterial survival ratio\%:

Bacterial survival ratio(\%) $=($ survivor bacterial count of experimental group $) /$ (bacteria count of control) $\times 100 \%$

The test of in vivo NIR irradiation enhanced antibacterial performance were carried out according to the previous reports, ${ }^{[8]]}$ and the details are available in the Materials and methods section of Supporting Information.

In Vitro Drug Release Study: For the controlled drug release behaviors test of HA-DA/rGO hydrogels, doxycycline was chosen as a model drug. ${ }^{[82,83]}$ To better explain the release mechanism of doxycycline from HA-DA/rGO hydrogels, the famous Korsmeyer-Peppas equation was used to fit with the release behaviors. The details of specific operation steps are available in the Materials and methods section of Supporting Information.

Antibacterial Activity of Doxycycline Encapsulated HA-DA/rGO Hydrogel: E. coli (gram-negative) and S. aureus (gram-positive) were chosen as the representative to evaluate the antibacterial activity of doxycycline-loaded HA-DA/rCO3 hydrogel by using a zone of inhibition test. ${ }^{[84,85]}$ The detail of the specific operation steps are available in the Materials and methods section of Supporting Information.

Hemolytic Activity Test of the Hydrogels: The test of in vitro hemolysis activity of these hydrogels was carried out according to ref. [86] The details of specific operation steps are available in the Materials and methods section of Supporting Information.

Cytocompatibility Evaluation of the Hydrogels: A direct contact test between L929 cells and hydrogels was used to measure the cytotoxicity of these HA-DA/rGO hydrogels based on the published reports. ${ }^{[73,87]}$ The details of specific operation steps are available in the Materials and methods section of Supporting Information.

In Vivo Wound Healing in a Full-Thickness Skin Defect Model: A mouse full-thickness wound model was carried out for the evaluation of the effect of HA-DA/rGO hydrogel on wound healing. All mice were randomly divided into four groups including Tegaderm film (control), hydrogel HA-DA/rGO0, hydrogel HA-DA/rGO3 and hydrogel HA-DA/ rGO3/Doxy. $7 \mathrm{~mm}$ diameter full thickness skin round wounds were created. The regeneration process of wounds was assessed by wound area monitoring. ${ }^{[88]}$ Collagen amount was evaluated by estimating hydroxylproline content using a commercial kit. ${ }^{[12]}$ The details are available in the Materials and methods section of Supporting Information.

Histology and Immunohistochemistry: Histology and immunohistochemistry examination was performed to evaluate blood vessels regeneration and inflammation. Then, the samples were immunohistochemistry stained with CD31 and TNF- $\alpha$ by a standard protocol. The details can be found in the Materials and methods section of Supporting Information.

Statistical Analysis: All the experimental data were statistically analyzed and the results were expressed as a mean \pm standard deviation (SD). Statistical differences were determined using one-way ANOVA followed by a Bonferroni post hoc test for multiple comparisons with SPSS, version 24 (IBM). In all cases, differences were considered significant if $P<0.05$.

\section{Supporting Information}

Supporting Information is available from the Wiley Online Library or from the author. 


\section{Acknowledgements}

Y.L. and X.Z. contributed equally to this work. This work was supported by the National Natural Science Foundation of China (grant number: 51673155), State Key Laboratory for Mechanical Behavior of Materials, the Fundamental Research Funds for the Central Universities, and the World-Class Universities (Disciplines) and the Characteristic Development Guidance Funds for the Central Universities.

\section{Conflict of Interest}

The authors declare no conflict of interest.

\section{Keywords}

adhesive conductive hydrogels, antioxidant, drug release, hemostat, wound healing

Received: January 4, 2019

Revised: February 4, 2019

Published online: February 20, 2019

[1] S. Bale, J. Cameron, S. Meaume, A. Ingegneri, in Science and Practice of Pressure Ulcer Management, Springer, London 2018, p. 111.

[2] N. Pazyar, R. Yaghoobi, E. Rafiee, A. Mehrabian, A. Feily, Skin Pharmacol. Physiol. 2014, 27, 303.

[3] S. Corcione, F. G. De Rosa, Curr. Opin. Infect. Dis. 2018, 31, 155.

[4] L. Rittié, J. Cell Commun. Signaling 2016, 10, 103.

[5] R. G. Rosique, M. J. Rosique, F. Junior, A. Jayme, Int. J. Inflammation 2015, 2015, 1

[6] M.-M. Yolanda, A.-V. Maria, F. Amaia, P. Marcos, P. Silvia, E. Dolores, O. Jesús, J. Stem Cell Res. Ther. 2014, 04, 2.

[7] H. Sorg, D. J. Tilkorn, S. Hager, J. Hauser, U. Mirastschijski, Eur. Surg. Res. 2017, 58, 81.

[8] R. F. Pereira, P. J. Bartolo, Adv. Wound Care 2016, 5, 208.

[9] X. Zhao, H. Wu, B. Guo, R. Dong, Y. Qiu, P. X. Ma, Biomaterials 2017, 122, 34

[10] E. A. Kamoun, E.-R. S. Kenawy, X. Chen, J. Adv. Res. 2017, 8, 217.

[11] H. Kim, J. Wound Manage. Res. 2018, 14, 141.

[12] J. Qu, X. Zhao, Y. Liang, T. Zhang, P. X. Ma, B. Guo, Biomaterials 2018, 183, 185.

[13] C. Ghobril, M. Grinstaff, Chem. Soc. Rev. 2015, 44, 1820.

[14] A. K. Gaharwar, N. A. Peppas, A. Khademhosseini, Biotechnol. Bioeng. 2014, 111, 441.

[15] P. J. Bouten, M. Zonjee, J. Bender, S. T. Yauw, H. van Goor, J. C. van Hest, R. Hoogenboom, Prog. Polym. Sci. 2014, 39, 1375.

[16] D. Naor, Front. Immunol. 2016, 7, 39.

[17] W. Shigeeda, M. Shibazaki, S. Yasuhira, T. Masuda, T. Tanita, Y. Kaneko, T. Sato, Y. Sekido, C. Maesawa, Oncotarget 2017, 8, 93729.

[18] D. Jiang, J. Liang, P. W. Noble, Annu. Rev. Cell Dev. Biol. 2007, 23, 435.

[19] W. J. Chen, G. Abatangelo, Wound Repair Regen. 1999, 7, 79.

[20] M. G. Neuman, R. M. Nanau, L. Oruña-Sanchez, G. Coto, J. Pharm. Pharm. Sci. 2015, 18, 53.

[21] T. Führmann, J. Obermeyer, C. Tator, M. Shoichet, Methods 2015, $84,60$.

[22] L. Li, N. Wang, X. Jin, R. Deng, S. Nie, L. Sun, Q. Wu, Y. Wei, C. Gong, Biomaterials 2014, 35, 3903.

[23] J. E. Greenwood, in Skin Tissue Engineering and Regenerative Medicine, (Eds: M. Z. Albanna, J. H. HolmesIV), Academic Press, Boston 2016, p. 185.
[24] K. L. Aya, R. Stern, Wound Repair Regener. 2014, 22, 579.

[25] J. H. Ryu, S. Hong, H. Lee, Acta Biomater. 2015, 27, 101.

[26] A. I. Neto, A. C. Cibrao, C. R. Correia, R. R. Carvalho, G. M. Luz, G. G. Ferrer, G. Botelho, C. Picart, N. M. Alves, J. F. Mano, Small 2014, 10, 2459.

[27] S. Hong, K. Yang, B. Kang, C. Lee, I. T. Song, E. Byun, K. I. Park, S. W. Cho, H. Lee, Adv. Funct. Mater. 2013, 23, 1774.

[28] P. Martin, Science 1997, 276, 75.

[29] S. A. Eming, P. Martin, M. Tomic-Canic, Sci. Transl. Med. 2014, 6, 265 sr6.

[30] B. Guo, A. Finne-Wistrand, A.-C. Albertsson, Biomacromolecules 2011, 12, 2601.

[31] B. Guo, L. Glavas, A.-C. Albertsson, Prog. Polym. Sci. 2013, 38, 1263.

[32] B. Guo, P. X. Ma, Biomacromolecules 2018, 19, 1764.

[33] L. Wang, Y. Wu, B. Guo, P. X. Ma, ACS Nano 2015, 9, 9167.

[34] Y. Wu, L. Wang, B. Guo, P. X. Ma, ACS Nano 2017, 11, 5646.

[35] M. Xie, L. Wang, J. Ge, B. Guo, P. X. Ma, ACS Appl. Mater. Interfaces 2015, 7, 6772.

[36] S. R. Shin, Y.-C. Li, H. L. Jang, P. Khoshakhlagh, M. Akbari, A. Nasajpour, Y. S. Zhang, A. Tamayol, A. Khademhosseini, Adv. Drug Delivery Rev. 2016, 105, 255.

[37] S. Goenka, V. Sant, S. Sant, J. Controlled Release 2014, 173, 75.

[38] O. C. Compton, S. T. Nguyen, Small 2010, 6, 711.

[39] M. Liu, J. Ji, X. Zhang, X. Zhang, B. Yang, F. Deng, Z. Li, K. Wang, Y. Yang, Y. Wei, J. Mater. Chem. B 2015, 3, 3476.

[40] N. Huang, S. Zhang, L. Yang, M. Liu, H. Li, Y. Zhang, S. Yao, ACS Appl. Mater. Interfaces 2015, 7, 17935.

[41] L. S. Nair, C. T. Laurencin, Prog. Polym. Sci. 2007, 32, 762.

[42] B. Guo, A. Finne-Wistrand, A.-C. Albertsson, Chem. Mater. 2011, 23, 1254.

[43] B. Guo, P. X. Ma, Sci. China: Chem. 2014, 57, 490.

[44] X. Zhao, M. Zhang, B. Guo, P. X. Ma, J. Mater. Chem. B 2016, 4, 6644.

[45] S. Zhong, Y. Zhang, C. Lim, Wiley Interdiscip. Rev.: Nanomed. Nanobiotechnol. 2010, 2, 510.

[46] N. Annabi, D. Rana, E. S. Sani, R. Portillo-Lara, J. L. Gifford, M. M. Fares, S. M. Mithieux, A. S. Weiss, Biomaterials 2017, 139, 229.

[47] X. Chen, Small Methods 2017, 1, 1600029.

[48] L. Li, J. Ge, B. Guo, P. X. Ma, Polym. Chem. 2014, 5, 2880.

[49] Y. Wu, B. Guo, P. X. Ma, ACS Macro Lett. 2014, 3, 1145.

[50] B. Guo, J. Qu, X. Zhao, M. Zhang, Acta Biomater. 2019, 84, 180.

[51] Z. Deng, T. Hu, Q. Lei, J. He, P. X. Ma, B. Guo, ACS Appl. Mater. Interfaces 2019, 11, 6796.

[52] J. Qu, X. Zhao, Y. Liang, Y. Xu, P. X. Ma, B. Guo, Chem. Eng. J. 2019, $362,548$.

[53] D. Gopinath, M. R. Ahmed, K. Gomathi, K. Chitra, P. K. Sehgal, R. Jayakumar, Biomaterials 2004, 25, 1911.

[54] V. Kant, A. Gopal, N. N. Pathak, P. Kumar, S. K. Tandan, D. Kumar, Int. Immunopharmacol. 2014, 20, 322.

[55] Y. Liang, X. Zhao, P. X. Ma, B. Guo, Y. Du, X. Han, J. Colloid Interface Sci. 2019, 536, 224.

[56] E. Lih, J. S. Lee, K. M. Park, K. D. Park, Acta Biomater. 2012, 8, 3261.

[57] S. H. Ku, J. Ryu, S. K. Hong, H. Lee, C. B. Park, Biomaterials 2010, $31,2535$.

[58] E. Pirila, N. Ramamurthy, P. Maisi, S. McClain, A. Kucine, J. Wahlgren, L. M. Golub, T. Salo, T. Sorsa, Curr. Med. Chem. 2001, 8, 281.

[59] D. D. Cissell, J. M. Link, J. C. Hu, K. A. Athanasiou, Tissue Eng., Part C 2017, 23, 243.

[60] M. B. Dreifke, A. A. Jayasuriya, A. C. Jayasuriya, Mater. Sci. Eng., C 2015, 48, 651.

[61] C. G. Decker, Y. Wang, S. J. Paluck, L. Shen, J. A. Loo, A. J. Levine, L. S. Miller, H. D. Maynard, Biomaterials 2016, 81, 157.

[62] C. L. Baum, C. J. Arpey, Dermatol. Surg. 2005, 31, 674. 
[63] R. L. Bronaugh, R. F. Stewart, E. R. Congdon, Toxicol. Appl. Pharmacol. 1982, 62, 481.

[64] B. Lu, T. Li, H. Zhao, X. Li, C. Gao, S. Zhang, E. Xie, Nanoscale 2012, 4, 2978.

[65] S. Barrientos, O. Stojadinovic, M. S. Golinko, H. Brem, M. Tomic-Canic, Wound Repair Regener. 2008, 16, 585.

[66] S. Barrientos, H. Brem, O. Stojadinovic, M. Tomic-Canic, Wound Repair Regener. 2014, 22, 569.

[67] H. H. Kim, J. B. Park, M. J. Kang, Y. H. Park, Int. J. Biol. Macromol. 2014, 70, 516.

[68] S. R. Shin, C. Zihlmann, M. Akbari, P. Assawes, L. Cheung, K. Zhang, V. Manoharan, Y. S. Zhang, M. Yuksekkaya, K. T. Wan, M. Nikkhah, M. R. Dokmeci, X. S. Tang, A. Khademhosseini, Small 2016, 12, 3677.

[69] L. Q. Xu, W. J. Yang, K.-G. Neoh, E.-T. Kang, G. D. Fu, Macromolecules 2010, 43, 8336.

[70] J. Jiang, L. Zhu, L. Zhu, B. Zhu, Y. Xu, Langmuir 2011, 27, 14180.

[71] Y. Wu, L. Wang, B. Guo, Y. Shao, P. X. Ma, Biomaterials 2016, 87, 18.

[72] Z. Deng, Y. Guo, X. Zhao, P. X. Ma, B. Guo, Chem. Mater. 2018, 30, 1729.

[73] X. Zhao, R. Dong, B. Guo, P. X. Ma, ACS Appl. Mater. Interfaces 2017, 9, 29595.

[74] J. Qu, X. Zhao, P. X. Ma, B. Guo, Acta Biomater. 2017, 58, 168.

[75] J. Qu, X. Zhao, P. X. Ma, B. Guo, Acta Biomater. 2018, 72, 55.

[76] X. Zhao, B. Guo, H. Wu, Y. Liang, P. X. Ma, Nat. Commun. 2018, 9, 2784.
[77] R. Gharibi, H. Yeganeh, A. Rezapour-Lactoee, Z. M. Hassan, ACS Appl. Mater. Interfaces 2015, 7, 24296.

[78] R. Dong, X. Zhao, B. Guo, P. X. Ma, ACS Appl. Mater. Interfaces 2016, 8, 17138.

[79] J. J. Bae, J. H. Yoon, S. Jeong, B. H. Moon, J. T. Han, H. J. Jeong, G.-W. Lee, H. R. Hwang, Y. H. Lee, S. Y. Jeong, Nanoscale 2015, 7, 15695.

[80] M.-C. Wu, A. R. Deokar, J.-H. Liao, P.-Y. Shih, Y.-C. Ling, ACS Nano 2013, 7, 1281 .

[81] H. Xu, Z. Fang, W. Tian, Y. Wang, Q. Ye, L. Zhang, J. Cai, Adv. Mater. 2018, 30, 1801100.

[82] C. J. Tormos, C. Abraham, S. V. Madihally, Drug Delivery Transl. Res. 2015, 5, 575.

[83] J. Zhao, X. Zhao, B. Guo, P. X. Ma, Biomacromolecules 2014, 15, 3246.

[84] S. J. Ryu, H. Jung, J. M. Oh, J. K. Lee, J. H. Choy, J. Phys. Chem. Solids 2010, 71, 685.

[85] C. Wiegand, M. Abel, P. Ruth, P. Elsner, U.-C. Hipler, J. Mate. Sci: Mater. Med. 2015, 26, 18.

[86] A. Sasidharan, L. S. Panchakarla, A. R. Sadanandan, A. Ashokan, P. Chandran, C. M. Girish, D. Menon, S. V. Nair, C. Rao, M. Koyakutty, Small 2012, 8, 1251.

[87] X. Zhao, P. Li, B. Guo, P. X. Ma, Acta Biomater. 2015, 26, 236.

[88] Z. Fan, B. Liu, J. Wang, S. Zhang, Q. Lin, P. Gong, L. Ma, S. Yang, Adv. Funct. Mater. 2014, 24, 3933. 